\title{
A Relational Framework for Classifier Engineering
}

\author{
Benny Kimelfeld \\ Technion - Israel Institute of Technology \\ Haifa 32000, Israel \\ bennyk@cs.technion.ac.il
}

\author{
Christopher Ré \\ Stanford University \\ Stanford, CA 94305, USA \\ chrismre@cs.stanford.edu
}

\begin{abstract}
In the design of analytical procedures and machine-learning solutions, a critical and time-consuming task is that of feature engineering, for which various recipes and tooling approaches have been developed. In this framework paper, we embark on the establishment of database foundations for feature engineering. We propose a formal framework for classification in the context of a relational database. The goal of this framework is to open the way to research and techniques to assist developers with the task of feature engineering by utilizing the database's modeling and understanding of data and queries, and by deploying the well studied principles of database management. As a first step, we demonstrate the usefulness of this framework by formally defining three key algorithmic challenges. The first challenge is that of separability, which is the problem of determining the existence of feature queries that agree with the training examples. The second is that of evaluating the $\mathrm{VC}$ dimension of the model class with respect to a given sequence of feature queries. The third challenge is identifiability, which is the task of testing for a property of independence among features that are represented as database queries. We give preliminary results on these challenges for the case where features are defined by means of conjunctive queries, and in particular we study the implication of various traditional syntactic restrictions on the inherent computational complexity.
\end{abstract}

\section{CCS Concepts}

-Information systems $\rightarrow$ Relational database model; Data analytics; •Computing methodologies $\rightarrow$ Feature selection; Supervised learning by classification; Supervised learning; •Theory of computation $\rightarrow$ Machine learning theory;

\section{Keywords}

Feature engineering; relational databases; machine learning; classifiers; conjunctive queries

Permission to make digital or hard copies of all or part of this work for personal or classroom use is granted without fee provided that copies are not made or distributed for profit or commercial advantage and that copies bear this notice and the full citation on the first page. Copyrights for components of this work owned by others than ACM must be honored. Abstracting with credit is permitted. To copy otherwise, or republish, to post on servers or to redistribute to lists, requires prior specific permission and/or a fee. Request permissions from permissions@ acm.org.

PODS'17, May 14-19, 2017, Chicago, Illinois, USA

(C) 2017 ACM. ISBN 978-1-4503-4198-1/17/05 . \$ $\$ 15.00$

DOI: http://dx.doi.org/10.1145/3034786.3034797

\section{INTRODUCTION}

In the design of analytics and machine-learning solutions, a critical and time-consuming task is feature engineering [24, 37]. Given its importance, recipes and tooling have been developed for practioners $[1,21]$. With the advent of frameworks like SAS, Cloudera's IBIS, Oracle's ORE, feature engineering is often carried out over relational data. Thus, a pressing challenge is to understand how to merge these new analytics with traditional database management techniques. In this paper we propose a relational framework for classification, a simple and popular analytic task.

\subsection{Feature Engineering}

The task of feature engineering is that of generating inputs (or signals) from available data, in order to improve the performance of the underlying model in solving a target problem. This target problem is typically classification (predict an unknown category of a given entity), or regression (predict the value of an unknown function over the entity). The model makes its prediction based on various properties, called features, of the given entity. We focus here on parametric models, where the model has a pre-determined structure with numerical parameters that are tuned by fitting into training examples, a process termed learning.

Naturally, the choice of features has a major impact on the resulting model. A suboptimal set of features may lead to overfitting (where the learned model does not well generalize beyond the training examples), or to underfitting (the model is incapable of capturing the target function due to lack of information or expressiveness) [21]. Another consideration is that of execution cost, as costly features may cast the model impractical [37]. There are also legal and moral considerations when decision makers are required to practice fairness and lack of discrimination $[14,36]$.

As an example, consider a scenario where the security branch of a credit-card company wishes to make an educated guess on whether an incoming transaction is an unauthorized purchase (i.e., made on a stolen card), and if so to take action such as inactivating the card and notifying the owner. To make such guess, the company uses its available information in the database, such as whether there were similar transactions in the past, whether the purchase is made in the same state of the owner's mailing address, or in the same country, the amount being paid, and so on. An employed engineer then selects a machine-learning library and learns a classifier, which can be trained based on past data on fraudulent activity. The classifier is simply a function that maps a vector of numbers into a yes/no decision; in turn, these num- 
bers, called features, encode relevant pieces of information (e.g., $f=+1 /-1$ depending on whether or not the transaction is in the country of the owner). The machine-learning library typically tunes parameters (or weights) of the classifier by fitting them to the examples. In our scenario, high quality is crucial: false positives disrupt legitimate business, and false negatives cause financial losses and customer disstress. So, the engineer produces additional features to consider by phrasing different questions (feature queries) about the transaction at stake, until she is satisfied with the results. This activity is referred to as feature engineering.

Analysts typically spend the bulk of their time on feature engineering $[20,24,37]$. This process includes a lot of trial and error, and stepwise addition or deletion of features [21]. We argue that involving the database semantics in feature engineering has the potential of automating some important tasks, and by that assist the engineer. For instance, the engineer may ask whether some class of simple feature queries (e.g., select-project-join) suffices to properly capture (i.e., achieve good classification of) the training data, or otherwise that more expressiveness is in need. This motivates the separability problem that we later discuss. She may also ask the complement question, which is whether the current set of feature queries is too detailed and allows the learning algorithm to overfit the examples and poorly generalize to future transactions. A traditional way to measure overfitting potential is to compute the Vapnik-Chervonenkis $(V C)$ dimension, and we later discuss the version of this problem in our setup, referred to as $V C$ dimensionality.

The engineer may also encounter the common technical challenge where the machine-learning library fails due to incompatible data, as it requires the matrix of training data to be of a full (column) degree. Partial degree (column dependence) may be an artifact of the training examples; but it may also be an inherent error in the feature design. As an example, for US owners the features "payment is in the US" "payment is in the owner's state" and "payment is in a different US state" have an inherent linear dependence among them: the first minus the second equals the third. We could use the database to detect such problems. This leads to the problem that we later refer to as identifiability.

We remark that the trend of deep learning [9] aims at eliminating the need for feature engineering by providing the model with sufficient expressive power to handle arbitrary raw data (or core features). This paradigm has a remarkable success, yet currently in a handful of domains [26].

\subsection{Proposed Framework}

The framework we propose aims to lead the way to novel research and techniques for utilizing the database's intimate understanding of raw data and queries, in order to fundamentally assist in the process of feature engineering. More precisely, our goal is to establish the first steps in a theory that embeds the automation of core tasks in the process of feature selection. After presenting the basic definitions, we illustrate the potential value of this framework by showing an analysis of tasks in the context of classification with features phrased as conjunctive queries; these tasks relate to underfitting (separability), overfitting ( $V C$ dimensionality), and algebraic coherence (identifiability).

Our framework is based on an entity schema, which is simply a relational schema with a distinguished relation symbol that represents entities. A database instance over an entity schema represents a collection of entities, along with additional (direct or indirect) knowledge about these entities. A feature query selects entities with a certain property, and a statistic is a sequence of feature queries. The central goal in classification is to train and apply a classifier (or a classifier model), which is a function that takes as input the statistic of an entity (i.e., the vector obtained by applying each feature query) and outputs a $+1 /-1$ decision. The task of training a classifier is the following: given a set of entities labeled with $+1 /-1$, produce a classifier from a predefined class of classifiers (the model class). A linear classifier, for example, is encoded as a vector of weights over the features in the statistic. In our framework, the collection of training examples is represented simply by an instance over the entity schema, along with a labeling function that maps each entity of the instance to $+1 /-1$.

For illustration, Figure 1(a) shows an entity schema $\mathbf{S}$ where entities are people (identified by a social security number), and Figure 1(b) shows a statistic $\Pi$ over $\mathbf{S}$ with two feature queries $\pi_{1}$ and $\pi_{2}$. Figure 2 shows the training workflow in our framework: an instance $I$ over $\mathbf{S}$ with a labeling of the entities (people) is transformed into a $(+1 /-1)$-matrix (consisting of the row of feature values for every entity), which in turn is used for building a classifier $h$. This classifier is then used for predicting the labels in a new instance, as illustrated in Figure 3.

The features, as defined above, are based on boolean properties of the entities: if the entity satisfies the property (i.e., it is "selected"), then the feature value is +1 , and otherwise -1 . Boolean features are highly important in practice, and in fact, we are aware of quite a few deployments where numerical values are translated into booleans ones (e.g., by means of bucketing, or binning, numbers into intervals). The more general case is where the feature query associates a numerical value with each entity. We defer the general case to future work, as our preliminary analysis applies to conjunctive queries, which are boolean, and we establish nontrivial results already there. Moreover, numerical values would substantially complicate the basic model, as we would need to argue about schema constraints that guarantee inclusion and functional dependencies in query answers (i.e., each feature associates a unique value with each entity); we find it an interesting and important aspect, but too far from the investigation of this paper.

After developing the framework, we turn to phrasing computational problems that are motivated by the practice of feature engineering where one aims to design a "good" statistic. We discuss three foundational problems for analytics that we can ask in this framework: separability - whether a perfect separator exists for a training set; identifiabilitytesting for a statistical property guaranteeing that the classifier model is uniquely defined given enough data; and $V C$ dimensionality - computing a fundamental measure of the complexity of a classifier class [34].

\subsection{Vision}

This framework paper aims to initiate a research effort of the theoretical database research community on the important task of feature engineering, and in particular, promote a future investigation that will contribute to this task the benefits of deep understanding of data and query fundamentals. Such an effort has already began in the system community of database research [37]. The preliminary results and anal- 
ysis we present do not aim to cover the ultimate use cases, but rather to instantiate and illustrate the vision in simple cases that we well understand (e.g., conjunctive queries). In particular, we focus on classification over boolean features that are represented as simple database queries that select entities. Future extensions should consider more expressive query languages, numerical features, and regression tasks beyond classification. (A discussion on possible future directions is in the concluding section.) Even in the restricted scope of this paper, our complexity results imply mainly intractability, and future work should seek tractable cases. Our results illustrate how the framework gives rise to relevant computational problems that one can formally define and explore, and these require some nontrivial analyses. Furthermore, the computational hardness emphasizes the need for fundamental research within the framework, which is what we hope to stir up.

\subsection{Organization}

The rest of the paper is organised as follows. In Section 2 we give preliminary definitions and notation. We present the framework in Section 3, and the associated computational problems in Section 4. Then, we investigate the problems of separability, VC dimensionality and identifiability for CQs in Sections 5, 6 and 7, respectively. We discuss related work in Section 8, and conclude in Section 9 where we also propose directions for future work.

\section{PRELIMINARIES}

In this section we give the basic definitions and terminology that we use throughout the paper.

\subsection{Relational Databases}

Our relational terminology is as follows. A schema is a collection of relation symbols. Each relation symbol $R$ has an associated arity $k$, and we use the conventional notation $R / k$ to compactly denote that $R$ is a relation symbol of arity $k$. We assume an infinite set Const of constants. An instance $I$ over a schema $\mathbf{S}$ associates with every $k$-ary relation symbol $R \in \mathbf{S}$ a finite subset of Const $^{k}$. We denote by $R^{I}$ the relation that $I$ associates with the relation symbol $R$. The active domain of an instance $I$, denoted adom $(I)$, is the set of all the constants in Const that are mentioned in the tuples of $I$. Two instances $I$ and $I^{\prime}$ are domain disjoint if $\operatorname{adom}(I)$ and $\operatorname{adom}\left(I^{\prime}\right)$ are disjoint. A fact over a schema $\mathbf{S}$ is an expression of the form $R\left(c_{1}, \ldots, c_{k}\right)$, where $R / k \in \mathbf{S}$ and $c_{1}, \ldots, c_{k}$ are constants. We say that the fact $R\left(c_{1}, \ldots, c_{k}\right)$ belongs to an instance $I$ over $\mathbf{S}$ if $R^{I}$ contains the tuple $\left(c_{1}, \ldots, c_{k}\right)$. By a slight abuse of notation, we view an instance as the set of its facts. So, for example, given two instances $I$ and $I^{\prime}$ over a schema $\mathbf{S}$, their union $I \cup I^{\prime}$ is the instance $J$ such that $R^{J}=R^{I} \cup R^{I^{\prime}}$ for all $R \in \mathbf{S}$.

Comment 2.1. While schema constraints are important for our framework, the results we later give are either oblivious to constraints or do not support them. Hence, to simplify the presentation we exclude constraints to begin with. Supporting constraints is an important future direction that we discuss in Section 9.

Let $I$ and $J$ be two instances over the same schema $\mathbf{S}$. A homomorphism from $I$ to $J$ is a mapping $\mu$ : adom $(I) \rightarrow$ $\operatorname{adom}(J)$ such that for every fact $f \in I$ we have $\mu(f) \in J$; here, $\mu(f)$ is the fact that is obtained from $f$ by replacing each constant $a$ with the constant $\mu(a)$. An endomorphism over $I$ is a homomorphism from $I$ to itself.

\subsection{Queries}

Let $\mathbf{S}$ be schema. A query (over $\mathbf{S}$ ) is a function $Q$ that is associated with an arity $k$, and that maps every instance $I$ over $\mathbf{S}$ into a finite subset $Q(I)$ of Const $^{k}$. A query $Q^{\prime}$ contains a query $Q$, in notation $Q \subseteq Q^{\prime}$, if $Q(I) \subseteq Q^{\prime}(I)$ for all instances $I$ over $\mathbf{S}$. If $Q \subseteq Q^{\prime}$ and $Q^{\prime} \subseteq Q$ then $Q$ and $Q^{\prime}$ are said to be equivalent. We will often be interested in unary queries $Q$ (i.e., where $k=1$ ); in that case we may abuse the notation and view $Q(I)$ as a set of constants $a$ rather than a set of tuples $(a)$.

In this paper we consider conjunctive queries without constants. Formally, here a Conjunctive Query (CQ) over a schema $\mathbf{S}$ is a logical formula $q(\mathbf{x})$ that has the form

$$
\exists \mathbf{y}\left[\phi_{1}(\mathbf{x}, \mathbf{y}) \wedge \cdots \wedge \phi_{m}(\mathbf{x}, \mathbf{y})\right]
$$

where $\mathbf{x}$ and $\mathbf{y}$ are disjoint sequences of variables and each $\phi_{i}$ is an atomic query over $\mathbf{S}$ (i.e., a formula that consists of a single relation symbol and no logical operators). ${ }^{1}$ The atomic formula $\phi_{i}$ is called an atom of $q$. We use the conventional notation of

$$
q(\mathbf{x}) \leftarrow \phi_{1}(\mathbf{x}, \mathbf{y}), \cdots, \phi_{m}(\mathbf{x}, \mathbf{y})
$$

to denote a CQ. We assume that the variables of $q$ come from a fixed, countably infinite set Vars, and that Const and Vars are disjoint. The left side $q(\mathbf{x})$ is called the head and the right side $\phi_{1}(\mathbf{x}, \mathbf{y}), \cdots, \phi_{m}(\mathbf{x}, \mathbf{y})$ is called the body. We require each variable in the head to occur at least once in the body. We may refer to a CQ by mentioning only its head $q(\mathbf{x})$ or even just $q$.

Let $\mathbf{S}$ be a schema, let $q$ be a CQ over $\mathbf{S}$, and let $I$ be an instance over $\mathbf{S}$. A homomorphism from $q$ to $I$ is a mapping from the variables of $q$ to adom $(I)$, such that for every atom $\phi$ of $q$, the fact $\mu(\phi)$ belongs to $I$; here, $\mu(\phi)$ is the fact that is obtained from $\phi$ by replacing each variable $z$ with the constant $\mu(z)$. The result of applying the CQ $q(\mathbf{x})$ to the instance $I$ is the relation that consists of all the tuples $\mu(\mathbf{x})$, where $\mu$ is a homomorphism from $q$ to $I$ and $\mu(\mathbf{x})$ is obtained from $\mathbf{x}$ by replacing every variable $x_{i}$ with $\mu\left(x_{i}\right)$. We denote this relation by $q(I)$.

The incidence graph [12] of a CQ $q$ is the bipartite graph that has the variables of $q$ on one side, the atoms on the other side, and an edge between an atom $\alpha$ and a variable $x$ whenever $\alpha$ contains $x$. We use $\mathcal{G}_{q}$ to denote the incidence graph of the CQ $q$.

We will consider CQs with special properties, which we define as follows. Consider a CQ $q$.

- $q$ is connected if $\mathcal{G}_{q}$ is connected.

- $q$ is self-join free if it does not have two distinct atoms with the same relation symbol.

We will also mention CQs with negation, that is, CQs of the form $q(\mathbf{x}) \leftarrow \phi_{1}(\mathbf{x}, \mathbf{y}), \cdots, \phi_{m}(\mathbf{x}, \mathbf{y})$ where each $\phi_{i}(\mathbf{x}, \mathbf{y})$ is either an atomic query (positive atom) or a negated atomic query (negative atom). We require such CQs to be safe in the sense that every variable occurs in at least one positive

\footnotetext{
${ }^{1}$ Note that in this paper CQs do not contain built-in relations such as $x>y$; we use this assumption in our analysis.
} 
Table 1: Main symbols

\begin{tabular}{c|l}
\hline$h$ & hypothesis/classifier $\{-1,1\}^{n} \rightarrow\{-1,1\}$ \\
$\mathbf{H}$ & hypothesis class \\
Lin & the class of linear classifiers \\
$\mathbf{S}$ & relational/entity schema \\
$\eta, \eta_{\mathbf{S}}$ & entity relation (unary) \\
$I, J$ & database instance \\
$\eta^{I}, \eta_{\mathbf{S}}^{I}$ & entity set of the instance $I$ \\
$e$ & entity in $\eta_{\mathbf{S}}^{I}$ \\
$\mathbf{Q L}$ & query language \\
$\mathbf{C Q}$ & the class of CQs (without constants) \\
$\pi$ & feature query (unary) \\
$\pi^{I}(e)$ & +1 if $e \in \pi(I)$ and -1 if $e \notin \pi(I)$ \\
$\Pi$ & statistic $\left(\pi_{1}, \ldots, \pi_{n}\right)$ \\
$\lambda$ & labeling function $\eta_{S}^{I} \rightarrow\{-1,1\}$ \\
\hline
\end{tabular}

atom. A negated atom has the classical (first-order-logic) semantics: the corresponding fact is not in the database instance. The definitions of connected and self-join free remain the same, while simply ignoring the negation symbols. Throughout the paper, we assume that CQs are without negation, unless explicitly stated otherwise.

We denote by CQ the class of CQs (as defined here, i.e., without constants), and by $\mathrm{CQ}\urcorner$ the class of CQs with negation. We also use the subscript sjf to denote the restriction to CQs without self joins, and the subscript con to denote the restriction to connected CQs. For example, $\mathrm{CQ}_{\mathrm{sjf}}$ is the class of CQs with negation and without self joins.

\subsection{Classifiers and Learning}

In this work, a classifier is a function of the form

$$
h:\{-1,1\}^{n} \rightarrow\{-1,1\}
$$

where $n$ is a natural number that we call the arity of $h$. A hypothesis class is a (possibly infinite) family $\mathbf{H}$ of classifiers, and a classifier in $\mathbf{H}$ is referred to as a hypothesis. We denote by $\mathbf{H}_{n}$ the restriction of $\mathbf{H}$ to the $n$-ary hypotheses in $\mathbf{H}$. An $n$-ary training collection is a multiset $T$ of pairs $\langle\mathbf{x}, y\rangle$ where $\mathbf{x} \in\{-1,1\}^{n}$ and $y \in\{-1,1\}$. We denote by $\mathbf{T}_{n}$ the class of all $n$-ary training collections. A cost function for a hypothesis class $\mathbf{H}$ is a function of the form

$$
c:\left(\cup_{n}\left(\mathbf{H}_{n} \times \mathbf{T}_{n}\right)\right) \rightarrow \mathbb{R}_{\geq 0}
$$

where $\mathbb{R}_{\geq 0}$ is the set of nonnegative numbers. Given a training collection $T$ and two hypotheses $h_{1}$ and $h_{2}$, the inequality $c\left(h_{1}, T\right)>c\left(h_{2}, T\right)$ implies that $h_{2}$ is preferred to $h_{1}$ according to $c$. In the context of a fixed hypothesis class $\mathbf{H}$ and a cost function $c$, learning a classifier is the task of finding a hypothesis $h \in \mathbf{H}_{n}$ that minimizes $c(h, T)$, given a training collection $T \in \mathbf{T}_{n}$.

It is importnat to allow $T$ to be a multiset in order to enable the scoring function to account for the frequency (rather than only existence) of examples. For the scope of this paper, though, being a multiset does not play any role, and the reader may view $T$ simply as a set.

We illustrate the above definitions on the important class of linear classifiers. An $n$-ary linear classifier is parameterized by a vector $\mathbf{w}=\left(w_{0}, \ldots, w_{n}\right) \in \mathbb{R}^{n+1}$, denoted by $\Lambda_{\mathbf{w}}$, and defined as follows for all $\mathbf{a} \in\{-1,1\}^{n}$.

$$
\Lambda_{\mathbf{w}}(\mathbf{a}) \stackrel{\text { def }}{=} \begin{cases}1 & \text { if } \mathbf{a} \cdot \mathbf{w}^{\prime} \geq w_{0} \\ -1 & \text { otherwise. }\end{cases}
$$

where $\mathbf{w}^{\prime}=\left(w_{1}, \ldots, w_{n}\right)$ and "." denotes the operation of dot product. By Lin we denote the class of linear classifiers. An example of a cost function is the least square cost that is given by

$$
l s q\left(\Lambda_{\mathbf{w}}, T\right) \stackrel{\text { def }}{=} \sum_{\langle\mathbf{x}, y\rangle \in T}\left(\mathbf{x} \cdot \mathbf{w}^{\prime}-w_{0}-y\right)^{2}
$$

for the arguments $\Lambda_{\mathbf{w}} \in \operatorname{Lin}_{n}$ and $T \in \mathbf{T}_{n}$.

More background on the basic theory of machine-learning classifiers, as well as the relevant linear algebra discussed in the next section, can be found in standard machine-learning textbooks, such as Shalev-Shwartz and Ben-David [31].

\subsection{Matrix Independence}

We denote by $\mathbf{0}^{n}$ the vector of $n$ zeroes, and by $\mathbf{1}^{n}$ the vector of $n$ ones. Let $M$ be an $n \times m$ real matrix (consisting of $n$ rows and $m$ columns). A linear column dependence in $M$ is a vector $\mathbf{w} \in \mathbb{R}^{m}$ such that $\mathbf{w} \neq \mathbf{0}^{m}$ and $M \cdot \mathbf{w}=\mathbf{0}^{n}$. A linear column dependence $\mathbf{w}$ in $M$ is an affine dependence in $M$ if $\mathbf{w} \cdot \mathbf{1}^{m}=0$ (i.e., the components of $\mathbf{w}$ sum up to 0 ). If $M$ does not have any linear column dependence, then we say that $M$ is linearly column independent. And if $M$ does not have any affine column dependence, then we say that $M$ is affinely column independent. Note that linear independence implies affine independence, but the other direction is not necessarily true.

\section{FRAMEWORK}

We now present our formal framework. A central notion in this framework is that of an entity schema, which is simply an ordinary relational schema with a distinguished relation symbol for representing entities. For simplicity, we assume that an entity is represented by a single constant (an identifier), hence the corresponding relation is unary. Formally, an entity schema is a pair $(\mathbf{S}, \eta)$, where $\mathbf{S}$ is a schema and $\eta$ is a unary relation symbol in $\mathbf{S}$. An instance over an entity schema $(\mathbf{S}, \eta)$ is simply an instance over $\mathbf{S}$. In the remainder of this paper all the schemas we consider are entity schemas. So, to simplify the presentation we refer to the entity schema $(\mathbf{S}, \eta)$ simply as $\mathbf{S}$, and refer to $\eta$ as $\eta_{\mathbf{S}}$.

Let $I$ be an instance over an entity schema $\mathbf{S}$. An entity of $I$ is a constant $a$ such that $\eta_{\mathbf{S}}(a) \in I$. Hence, $I$ represents a set of entities along with information about the entities; this information is contained in the remaining relations, which can be joined with $\eta_{\mathbf{S}}$. By a slight abuse of notation, we use $\eta_{\mathrm{S}}^{I}$ to represent the set of all the entities of $I$.

EXAMPLE 3.1. We use a running example that instantiates the credit-card scenario from the introduction. Figure 1(a) depicts the entity schema $\mathbf{S}$ of the example, with one unary relation $\mathbf{T} x \mathrm{n}$, which is $\eta_{\mathbf{S}}$, and two quaternary relations Txnlnfo and Card. The box on the top of Figure 3 contains an instance $I^{\prime}$ over $\mathbf{S}$. The entities are the transaction identifiers 5, 6 and 7 , and these are the members of $\eta_{\mathbf{S}}^{I^{\prime}}=\{5,6,7\}$

Let $\mathbf{S}$ be an entity schema, and let $I$ be an instance over $\mathbf{S}$. A feature query (over $\mathbf{S}$ ) is a unary query $\pi$ over the schema 


\begin{tabular}{|c|c|c|c|c|}
\hline $\mathrm{T} \times \mathrm{n}=\eta_{\mathbf{S}}$ & \multicolumn{4}{|c|}{ Txnlnfo } \\
\hline$i d$ & $t x n$ & card & country & state \\
\hline \multicolumn{5}{|c|}{ Card } \\
\hline$i d$ & $s s n$ & country & state & \\
\hline
\end{tabular}

(a) An entity schema $\mathbf{S}$

$$
\begin{aligned}
& \pi_{1}(s) \leftarrow \operatorname{TxnInfo}(t, n, c, s), \operatorname{Card}(n, c, s) \\
& \pi_{2}(s) \leftarrow \operatorname{Txnlnfo}(t, n, c, s), \operatorname{Card}\left(n, c, s^{\prime}\right)
\end{aligned}
$$

(b) A statistic $\Pi=\left(\pi_{1}, \pi_{2}\right)$ over $\mathbf{S}$

\begin{tabular}{|c|c|c|c|c|c|}
\hline \multirow{2}{*}{$\frac{\mathrm{Txn}}{i d}$} & \multirow[b]{2}{*}{$\lambda$} & \multicolumn{4}{|c|}{ Txnlnfo } \\
\hline & & $t x n$ & card & country & state \\
\hline 1 & 1 & 1 & 100 & US & $\mathrm{GA}$ \\
\hline 2 & 1 & 2 & 100 & US & NY \\
\hline 3 & -1 & 3 & 101 & $\mathrm{BR}$ & RJ \\
\hline 4 & 1 & 4 & 102 & US & $\mathrm{CA}$ \\
\hline \multicolumn{6}{|c|}{ Card } \\
\hline & $i d$ & ssn & country & state & \\
\hline & 100 & 200 & US & GA & \\
\hline & 101 & 201 & US & FL & \\
\hline & 102 & 202 & $\mathrm{BR}$ & SP & \\
\hline
\end{tabular}

Figure 1: An entity schema and a statistic

Training instance $(I, \lambda)$

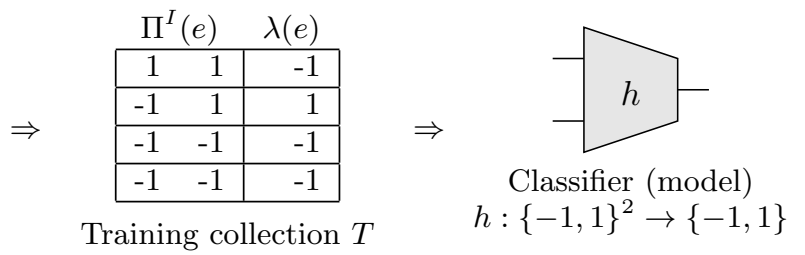

Figure 2: The training process

S. We will always be interested in only the entities that are selected by a feature query, and we will be interested in cases where $\pi$ is represented in a query language QL (e.g., $\mathrm{CQ}$ ); we then say that $\pi$ is in $\mathbf{Q L}$.

EXAMPLE 3.2. Figure 1(b) shows two feature queries in CQ: $\pi_{1}$ selects all transactions that took place in the same country and state of the owner's maling address, and $\pi_{2}$ selects all the ones that took place in the same country (but not necessarily same state) of the owner.

Let $\pi$ be a feature query over an entity schema $\mathbf{S}$. We say that $\pi$ is selective on an entity instance $I$ if at least one entity of $I$ is missing from $\pi(I)$. We say that $\pi$ is selective if it is selective on at least one instance over $\mathbf{S}$ (or in other words, $\pi$ is not logically contained in $\eta_{\mathbf{S}}$ ).

Let $\mathbf{S}$ be an entity schema, let $I$ be an instance over $\mathbf{S}$, and let $\pi$ be a feature query. We denote by $\pi^{I}$ the function from $\eta_{\mathbf{S}}^{I}$ to $\{-1,1\}$ where

\begin{tabular}{|c|c|c|c|c|c|}
\hline \multirow{2}{*}{$\frac{\mathrm{Txn}}{i d}$} & \multicolumn{5}{|c|}{ Txnlnfo } \\
\hline & & card & \multicolumn{2}{|c|}{ country } & state \\
\hline 5 & & 105 & \multicolumn{2}{|c|}{ US } & AK \\
\hline 6 & & 105 & \multicolumn{2}{|c|}{ US } & NY \\
\hline 7 & & 110 & \multicolumn{2}{|c|}{$\mathrm{BR}$} & $\mathrm{RJ}$ \\
\hline \multicolumn{6}{|c|}{ Card } \\
\hline & & \multicolumn{2}{|c|}{ country } & \multicolumn{2}{|c|}{ state } \\
\hline & & \multicolumn{2}{|c|}{ US } & \multicolumn{2}{|c|}{$\mathrm{AK}$} \\
\hline & & \multicolumn{2}{|c|}{$\mathrm{BR}$} & $\mathrm{SF}$ & \\
\hline
\end{tabular}

$$
\pi^{I}(e s)= \begin{cases}1 & \text { if } e \in \pi(I) \\ -1 & \text { otherwise }\end{cases}
$$

Instance $I^{\prime}$ over $\mathbf{S}$

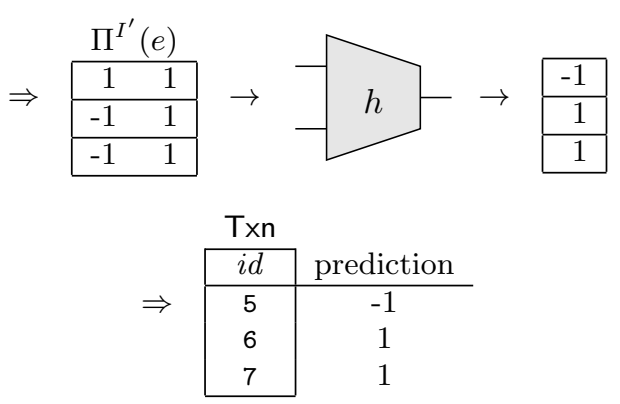

Figure 3: The prediction process

Let $\mathbf{S}$ be an entity schema. A statistic (over $\mathbf{S}$ ) is a sequence $\Pi=\left(\pi_{1}, \ldots, \pi_{n}\right)$ of feature queries. We say that $\Pi$ is in a query language QL if each $\pi_{i}$ is in QL. Given an instance $I$ over $\mathbf{S}$, we denote by $\Pi^{I}$ the function $\left(\pi_{1}^{I}, \ldots, \pi_{n}^{I}\right)$ from $\eta_{\mathbf{S}}^{I}$ to $\{-1,1\}^{n}$ that maps every entity $e \in \eta_{\mathbf{S}}^{I}$ to the sequence $\left(\pi_{1}^{I}(e), \ldots, \pi_{n}^{I}(e)\right)$.

EXAmple 3.3. Figure 1 (b) shows the statistic $\Pi=\left(\pi_{1}, \pi_{2}\right)$ over the schema $\mathbf{S}$ of Figure 1(a). The middle layer of Figure 3 contains (on its left) the tuples $\Pi^{I^{\prime}}(e)$ for the entities $e$ in the instance $I^{\prime}$ of the top box in this figure. For example, the top row corresponds to $\Pi^{I^{\prime}}(5)=(1,1)$, which is due to the fact Transaction 5 took place in the same country and state of the card holder.

Let $\mathbf{S}$ be an entity schema. A labeling of an instance $I$ over $\mathbf{S}$ is a function

$$
\lambda: \eta_{\mathbf{S}}^{I} \rightarrow\{-1,1\}
$$

that partitions the entities into negative examples (i.e., $e$ where $\lambda(e)=-1$ ) and positive examples (i.e., $e$ where $\lambda(e)=$ 1). A training instance over $\mathbf{S}$ is a pair $(I, \lambda)$, where $I$ is an instance over $\mathbf{S}$ and $\lambda$ is a labeling of $I$.

Given an entity schema $\mathbf{S}$, a statistic $\Pi$ and a training instance $(I, \lambda)$ together define a training collection, namely, the one that consists of the tuple $\left\langle\Pi^{I}(e), \lambda(e)\right\rangle$ for every entity $e \in \eta_{\mathbf{S}}^{I}$.

ExAmple 3.4. Continuing our running example, Figure 2 shows a training instance $(I, \lambda)$ over the entity schema $\mathbf{S}$ of Figure 1(a), where $\lambda$ is represented in the Txn relation. With the statistic $\Pi$ of Figure 1(b) we then get the training collection in the left bottom part of Figure 2. From this training instance a classifier $h$ is learned, and is applied for prediction on future instances, as illustrated in Figure 3 for the instance $I^{\prime}$ that we referred to in the previous examples. 


\section{COMPUTATIONAL PROBLEMS}

We now describe three computational problems that are motivated by the design of machine-learning solutions, and in particular feature engineering.

\subsection{Separability}

Separability is perhaps the most basic notion of learning. The traditional presentation of learning theory typically begins with the "noise free" case where the labeled examples are required to be perfectly separated by the features. In our framework separability refers to the following task: given a training instance over an entity schema, determine whether there is statistic and a classifier that agree with (i.e., classify precisely as) the example labels. It is, of course, a simplification of the practically motivated problem, where some noise is allowed (and say, $(1-\epsilon)$ of the examples are required to be correctly satisfied). We adopt the simplified (textbook) task as a first step, and show that it already leads to nontrivial insights within our framework.

The problem is parameterized by two important components: the family of classifiers in consideration, and the query language used for phrasing feature queries. The formal defintion of the problem is as follows.

Let $\mathbf{S}$ be a schema, $\Pi$ a statistic over $\mathbf{S}$, and $\mathbf{H}$ a hypothesis class. A training instance $(I, \lambda)$ is $\mathbf{H}$-separable with respect to (w.r.t.) $\Pi$ if there exists a hypothesis $h \in \mathbf{H}$ that fully agrees with $\lambda$; that is, $h$ and $\Pi$ have the same arity, and $h\left(\Pi^{I}(e)\right)=\lambda(e)$ for every $e \in \eta_{\mathbf{S}}^{I}$.

Problem 1 (Separability). For a hypothesis class $\mathbf{H}$ and a query language $\mathbf{Q L}$, the problem $(\mathbf{H}, \mathbf{Q L})$-separability is the following. Given an entity schema $\mathbf{S}$ and a training instance $(I, \lambda)$ over $\mathbf{S}$, determine whether there exists a statistic $\Pi$ in $\mathbf{Q L}$ such that $(I, \lambda)$ is $\mathbf{H}$-separable w.r.t. $\Pi$.

ExAMPlE 4.1. We continue with our running example, and consider the training instance $(I, \lambda)$ of Figure 2. Suppose that $\mathbf{H}$ is the class Lin of linear classifiers, and that QL is the class CQ. Then $(I, \lambda)$ is a "yes" instance of the separability problem, and a witness is the statistic $\Pi=\left(\pi_{1}, \pi_{2}\right)$ of Figure $1(\mathrm{~b})$ with the classifier $\pi_{2}-\pi_{1} \geq 1$. Now suppose that we add an entity 5 , the tuple $(5,102$, US, AL) to Txnlnfo, and the labeling $\lambda(5)=-1$. The new training instance then becomes a "no" instance of the separability problem since, intuitively, there is no way to distinguish between 4 and 5 using QL over $I$, and yet, $\lambda$ labels 4 and 5 differently. We make this point precise in Section 5 .

We remark that a problem similar to separability is that of separating graphs by tree patterns $[13,25]$.

\subsection{VC Dimensionality}

The Vapnik-Chervonenkis ( $V C$ ) dimension is a measure of complexity of a hypothesis class, and is a de facto complexity measure for learnability. Bounds for generalization (how well a learned classifier does on unseen data) typically depend on the VC dimension. It measures the capacity of the classifier class, and is a key indicator of how much data one needs to reliably train the classifier: if this amount is low with respect to the $\mathrm{VC}$ dimension, then the classifier may overfit. If the amount of training data is high with respect to the $\mathrm{VC}$ dimension, we may be missing opportunities to devise a more accurate classifier.
As an example, the class of polynomial classifiers is more expressive than that of the linear classifiers, so there is a higher capability of a polynomial-classifier learner to overfit (i.e., exploit specific properties that are exhibited scarcely in the training examples but are not representative of the general population). Similarly, a deep decision tree might be constructed to handle every individual example, while a shallow one will have to utilize common properties, and hence, intuitively, to better apply to the behavior in the general population. VC dimension is a mathematical measure that aims to capture this expressive power in a manner that is uniform across model classes. In particular, high VC dimension implies a complicated classifier space that has a high ability to overfit its training data, and so, a high volume of training data is required for effective learning.

In our framework, VC dimension is not only a function of the hypothesis class, but also that of the statistic that translates entities into feature vectors. We give the formal definition next.

Let $\mathbf{S}$ be a schema, $\Pi$ a statistic over $\mathbf{S}$, and $\mathbf{H}$ a hypothesis class. An instance $I$ over $\mathbf{S}$ is shattered by $\mathbf{H}$ w.r.t. $\Pi$ if for every labeling $\lambda$ of $I$ there exists a hypothesis $h \in \mathbf{H}$ that fully agrees with $\lambda$; that is, $h$ and $\Pi$ have the same arity, and $h\left(\Pi^{I}(e)\right)=\lambda(e)$ for every $e \in \eta_{\mathbf{S}}^{I}$.

The $V C$ dimension of $\mathbf{H}$ w.r.t. $\Pi$ is the maximal number $m$ such that there exists an instance $I$ over $\mathbf{S}$ where $I$ has $m$ entities and $I$ is shattered by $\mathbf{H}$ w.r.t. $\Pi$. The dimensionality problem is defined as follows.

Problem 2 (Dimensionality). Let $\mathbf{H}$ be a hypothesis class and $\mathbf{Q L}$ a query language. The computational problem (H, QL)-dimensionality is the following. Given an entity schema $\mathbf{S}$ and a statistic $\Pi$ in $\mathbf{Q L}$, compute the $V C$ dimension of $\mathbf{H}$ w.r.t. $\Pi$.

ExAmple 4.2. Recall $\mathbf{S}$ and $\Pi$ of our running example (Figure 1). Computing the VC dimension of Lin w.r.t. $\Pi$ is an instance of ( $\operatorname{Lin}, \mathrm{CQ}$ )-dimensionality. Our results in Section 6 show that this dimension is 3 . Hence, there exists an instance $I$ with three entities, such that we can find a perfect linear classifier for every labeling $\lambda$ for $I$. Yet, no such instance exists with four or more entities.

\subsection{Statistic Identifiability}

Identifiability is a classical task that asks whether it is possible for one to learn the parameters of the given classifier model unambiguously from some data set. Here, the question refers to a given statistic, and we consider the case where training is done by means of optimization via linear algebra; we ask whether the space of solutions is bounded. More formally, this problem boils down to deciding, given a statistic, whether there exists any training instance such that the resulting feature matrix is of full column dimension (i.e., the columns are linearly independent). We also consider the variant where linear independence is relaxed to affine independence. (See Section 2 for the formal definitions.) Next, we give the formal definition.

Let $\mathbf{S}$ be an entity schema, $\Pi$ a statistic over $\mathbf{S}$, and $I$ an instance of $\mathbf{S}$. We fix an arbitrary order over the entities of $I$, and denote by $\llbracket \Pi^{I} \rrbracket$ the matrix that consists of the rows $\Pi^{I}(e)$ for every $e \in \eta_{\mathbf{S}}^{I}$ in order. We say that $\Pi$ is linearly identifiable if there exists an instance $I$ over $\mathbf{S}$ such that the matrix $\llbracket \Pi^{I} \rrbracket$ is linearly column independent. We say that $\Pi$ is affinely identifiable if there exists an instance $I$ over $\mathbf{S}$ such 
that the matrix $\llbracket \Pi^{I} \rrbracket$ is affinely column independent. Recall that if $\llbracket \Pi^{I} \rrbracket$ is linearly independent, then it is also affinely independent. Hence, we get the following fact, which we record here for later reference.

FACT 4.3. Let $\Pi$ be a statistic. If $\Pi$ is linearly identifiable, then $\Pi$ is affinely identifiable.

Both types of identifiability are important properties in the design of machine-learning solutions [27]. Particularly, in the case of the hypothesis class Lin and the cost function $l s q$ (defined in Section 2.3), linear independence implies that there is a single optimal hypothesis, whereas its absence implies that the space of optimal solutions is unbounded. Affine independence likewise arises in different cost functions such as maximum entropy [35]. The corresponding computational problem is formally defined as follows.

Problem 3 (IDEntifiability). Let $\mathbf{Q L}$ be a query language. The computational problem of linear (respectively, affine) QL-identifiability is that of testing, given an entity schema $\mathbf{S}$ and a statistic $\Pi$ over $\mathbf{S}$, whether $\Pi$ is linearly (respectively, affinely) identifiable.

Example 4.4. Consider again $\mathbf{S}$ and $\Pi$ of our running example (Figure 1). Then $\mathbf{S}$ and $\Pi$ form a "yes" instance of the linear (and affine) CQ-identifiability problem. Indeed, $\Pi$ is linearly (and affinely) identifiable, and a witness instance is the instance $I$ of Figure 2 with Txn restricted to the entities 1 and 2 (or 3 and 2, but not 1 and 3). In Section 7 we will show that under certain conditions (that hold in our case), a statistic that consists of CQ feature queries is always identifiable, unless two or more of the feature queries are equivalent.

\section{SEPARABILITY W.R.T. CQS}

We now discuss the complexity of separability in the case where feature queries are from the class of CQs. We first state the complexity results of this section.

\subsection{Complexity Results}

Our first result states coNP-hardness in the case where feature queries are CQs without self joins, and the hypothesis class is that of linear classifiers. For illustration, we refer the reader to the "yes" and "no" instances of Example 4.1.

THEOREM 5.1. (Lin, CQ)-separability is a coNP-complete problem. Moreover, there exists a fixed entity schema $\mathbf{S}$ such that (Lin, CQ)-separability is coNP-hard over $\mathbf{S}$.

We prove the theorem later in this section. In the proof of hardness we construct CQs over a fixed schema, but self joins are allowed. Next, we consider the case of self-joinfree CQs. Interestingly, restricting the problem to simpler CQs (by disallowing self joins) does not make the problem easier; in fact, under conventional complexity assumptions it is harder!

\section{THEOREM 5.2. ( $\left(\right.$ in, $\left.\mathbf{Q L}_{\mathrm{sjf}}\right)$-separability is $\Sigma_{2}^{\mathrm{P}}$-complete.}

The proof of Theorem 5.2 is in the appendix. Intuitively, the reason for the increased complexity is that self joins allow us to (efficiently) formulate a single statistic $\Pi$ of representative ("canonical") feature CQs that captures the entire space of statistics; that is, if any statistic provides separation, then so does $\Pi$. In particular, with self joins the problem boils down to deciding on the existence of a homomorphism. Yet, without self joins it appears that we cannot do better than to inspect an exponential space of statistics, and solve the homomorphism problem in each. Finally, we remark that fixing the schema $\mathbf{S}$ in the case of $\mathbf{Q} \mathbf{L}_{\mathrm{sjf}}$ would make the separability problem solvable in polynomial time, since the number of possible statistics (without equivalent feature queries) is bounded by a fixed constant, and each feature query can be evaluated in polynomial time.

Comment 5.3. For CQs with constants, the separability problem is trivial and not interesting, since the positive examples can be hardcoded into (i.e., completely overfitted by) the statistic. In Figure 2, for instance, we could encode each of the first, second, and third tuples of Txnlnfo (and even their join with Card) in a CQ that selects precisely the corresponding transactions. It would, however, be interesting to enforce restrictions on the usage of constants (e.g., limit their number), and we leave that for future work. (See further discussion in Section 9.)

\subsection{Proof of Theorem $\mathbf{5 . 1}$}

We now prove Theorem 5.1. We need to prove membership in coNP and coNP-hardness.

The easier part is the proof of hardness, and it is given in the appendix. The proof constructs a reduction from the maximum-clique problem over graphs. Our fixed schema is $\mathbf{S}_{0}$ and it consists of the relation symbols $G / 3$ and $\eta / 1$, where $\eta_{\mathbf{S}}=\eta$. The lemma applies to every hypothesis class $\mathbf{H}$ (including Lin), under the very basic assumption that $\mathbf{H}$ contains the unary classifier $h(x)=x$; in that case we say that $\mathbf{H}$ allows copying.

LeMma 5.4. Let $\mathbf{H}$ be a hypothesis class that allows copying. The problem (H, CQ)-separability is coNP-hard already over the schema $\mathbf{S}_{0}$ and for two entities.

We now turn to proving membership in coNP. Let $I$ be an instance of an entity schema $\mathbf{S}$. Define a unique variable $x_{c}$ for every constant $c$ in adom $(I)$. For every fact $f$ of $I$, let $\phi_{f}$ denote the atomic formula obtained from $f$ by replacing every constant $c$ with the variable $x_{c}$. Let $\Phi_{I}$ denote the conjunction of the atoms $\phi_{f}$ over all facts $f$ in $I$. For an entity $e$ of $I$, the canonical $C Q$ of $e$ (w.r.t. $I$ ), denoted $Q_{e}^{I}$, is the CQ defined by $Q_{e}^{I}\left(x_{e}\right) \leftarrow \Phi_{I}$. Following is an easy proposition.

Proposition 5.5. Let $\mathbf{S}$ be an entity schema, I an instance of $\mathbf{S}$, and $e$ and $e^{\prime}$ be two entities of I. The following are equivalent.

$$
\begin{aligned}
& \text { 1. } e^{\prime} \in Q_{e}^{I}(I) \text {. } \\
& \text { 2. For every feature query } \pi \text { in } \mathrm{CQ} \text {, if } e \in \pi(I) \text { then } e^{\prime} \in \\
& \pi(I) \text {. }
\end{aligned}
$$

Let $I$ be an instance of an entity schema $\mathbf{S}$. We denote by $\preceq_{I}$ the binary relation over $\eta_{\mathbf{S}}^{I}$ such that $e \preceq_{I} e^{\prime}$ if and only if $e^{\prime} \in Q_{e}^{I}(I)$. The following proposition follows immediately from Proposition 5.5.

Proposition 5.6. Let $\mathbf{S}$ be an entity schema, and $I$ an instance of $\mathbf{S}$. The order $\preceq_{I}$ is a preorder, that is, $\preceq_{I}$ is reflexive and transitive. 
Let $(I, \lambda)$ be a training instance of an entity schema $\mathbf{S}$. Recall that an equivalence class of a preorder $\preceq$ is an equivalence class of the equivalence relation " $x \preceq y$ and $y \preceq x$." In the case of $\preceq_{I}$, an equivalence class $E$ is a set of entities.

Proposition 5.7. Let $\mathbf{S}$ be an entity schema, and $I$ an instance of $\mathbf{S}$. Let $E_{1}, \ldots, E_{m}$ be the equivalence classes of the preorder $\preceq_{I}$. There is a statistic $\Pi=\left(\pi_{1}, \ldots, \pi_{m}\right)$ in $\mathrm{CQ}$ with the following properties.

- Every entity $e \in E_{i}$ satisfies $e \in \pi_{i}(I)$.

- $e \notin \pi_{j}(I)$ for all $i$ and $j$ such that $1 \leq i<j \leq m$ and $e \in E_{i}$.

Proof. For each $E_{i}$, select a member entity $e_{i}$ and denote by $\pi_{i}^{\prime}$ the CQ $Q_{e_{i}}^{I}(I)$. We extend $\preceq_{I}$ to the CQs $\pi_{i}^{\prime}$ naturally, by defining $\pi_{i}^{\prime} \preceq_{I} \pi_{j}^{\prime}$ if and only if $e_{i} \preceq_{I} e_{j}$. Observe that $\preceq_{I}$ is a partial order over the $\pi_{i}^{\prime}$, and in particular, $\preceq_{I}$ is acyclic. We then take as $\pi_{1}, \ldots, \pi_{m}$ the sequence that consists of $\pi_{1}^{\prime}, \ldots, \pi_{m}^{\prime}$, sorted in a topological order according to $\preceq_{I}$; that is, if $i \neq j$ and $\pi_{i} \preceq_{I} \pi_{j}$, then $i<j$.

An equivalence class $E$ of $\preceq_{I}$ is said to be consistent with $\lambda$ if $\lambda(e)=\lambda\left(e^{\prime}\right)$ for every two entities $e$ and $e^{\prime}$ in $E$. Following is a key lemma for this section.

LEMMA 5.8. Let $(I, \lambda)$ be a training instance of an entity schema $\mathbf{S}$. The following are equivalent.

1. There exists a statistic $\Pi$ in $\mathrm{CQ}$ such that $(I, \lambda)$ is Linseparable w.r.t. $\Pi$.

2. Every equivalence class of $\preceq_{I}$ is consistent with $\lambda$.

ProOF. We prove each direction separately.

$\mathbf{1} \rightarrow \mathbf{2}$. This direction is a straightforward consequence of Proposition 5.5. In particular, if $e$ and $e^{\prime}$ are two entities such that $e \preceq_{I} e^{\prime}$ and $e^{\prime} \preceq_{I} e$, then from Proposition 5.5 it follows that every statistic $\Pi$ in CQ gives precisely the same feature values for $e$ and $e^{\prime}$, and in particular, if $\lambda(e) \neq \lambda\left(e^{\prime}\right)$ then $\lambda$ is not $\mathbf{H}$-separable w.r.t. $\Pi$.

$\mathbf{2} \rightarrow \mathbf{1}$. We now assume that every equivalence class of $\preceq_{I}$ is consistent with $\lambda$. Let $\Pi=\left(\pi_{1}, \ldots, \pi_{m}\right)$ be the statistic of Proposition 5.7. We will construct a linear classifier $h$ over $\Pi$, such that $h$ perfectly fits $\lambda$. Hence, the existence of $\Pi$ and $h$ provides the required proof. The classifier $h$ is defined as

$$
\delta_{1}+\cdots+\delta_{m} \geq 0
$$

where the $\delta_{j}$ are defined as follows for $j=1, \ldots, m$ :

$$
\delta_{j} \stackrel{\text { def }}{=} w_{j} \cdot\left(1+\pi_{j}^{I}\right)
$$

where:

$$
w_{j} \stackrel{\text { def }}{=} \frac{1}{2}\left(\lambda\left(e_{j}\right)-\sum_{k=1}^{j-1} \delta_{k}\left(e_{j}\right)\right)
$$

To complete the proof, we need to prove that $h$ classifies every entity $e$ according to $\lambda$. Let $e \in \eta_{\mathbf{S}}(I)$ be given, and suppose that $e$ belongs to $E_{i}$. Then, by assumption we have $\lambda(e)=\lambda\left(e_{i}\right)$. Moreover, due to Proposition 5.5 we have $\Pi(e)=\Pi\left(e_{i}\right)$. Therefore, it suffices to prove that $h$ classifies $e_{i}$ according to $\lambda$. First, we break the sum $\sum_{j=1}^{m} \delta_{j}\left(e_{i}\right)$.

$$
\sum_{j=1}^{m} \delta_{j}\left(e_{i}\right)=\left(\sum_{j=1}^{i-1} \delta_{j}\left(e_{i}\right)\right)+\delta_{i}\left(e_{i}\right)+\left(\sum_{j=i+1}^{m} \delta_{j}\left(e_{i}\right)\right)
$$

We have the following.

$$
\begin{aligned}
\delta_{i}\left(e_{i}\right) & =w_{i} \cdot\left(1+\pi_{i}^{I}\left(e_{i}\right)\right)=w_{i} \cdot(1+1)=2 w_{i} \\
& =\lambda\left(e_{i}\right)-\left(\sum_{j=1}^{i-1} \delta_{j}\left(e_{i}\right)\right)
\end{aligned}
$$

Moreover, as $\Pi$ is the statistic of Proposition 5.7, for $j>i$ we have $\pi_{j}^{I}\left(e_{i}\right)=-1$, and therefore,

$$
\delta_{j}\left(e_{i}\right)=w_{i} \cdot(1-1)=0 .
$$

Hence, from (1) and (2) we get the following.

$$
\sum_{j=1}^{m} \delta_{j}\left(e_{i}\right)=\left(\sum_{j=1}^{i-1} \delta_{j}\left(e_{i}\right)\right)+\lambda\left(e_{i}\right)-\left(\sum_{j=1}^{i-1} \delta_{j}\left(e_{i}\right)\right)=\lambda\left(e_{i}\right)
$$

In particular we have $\sum_{j=1}^{m} \delta_{j}\left(e_{i}\right)>0$ when $\lambda\left(e_{i}\right)=1$, and $\sum_{j=1}^{m} \delta_{j}\left(e_{i}\right)<0$ when $\lambda\left(e_{i}\right)=-1$, as required.

From Lemma 5.8 we conclude the required membership in coNP.

\section{LEMma 5.9. (Lin, CQ)-separability is in coNP.}

Proof. From Lemma 5.8 we conclude that as an evidence of non-separability it suffices to show two entities $e$ and $e^{\prime}$ with $\lambda(e) \neq \lambda\left(e^{\prime}\right)$, a homomorphism $\mu_{1}$ from $Q_{e}^{I}$ to $I$ with $\mu_{1}\left(x_{e}\right)=e^{\prime}$, and a homomorphism $\mu_{2}$ from $Q_{e^{\prime}}^{I}$ to $I$ with $\mu_{2}\left(x_{e^{\prime}}\right)=e$.

\section{VC DIMENSIONALITY W.R.T. CQS}

Next, we investigate the problem of VC dimensionality for the query language of connected CQs, and the class of linear classifiers. Intuitively, the need for connectivity is due to the fact that with disconnected CQs, it may be the case that CQs are not equivalent, but they cannot distinguish between entities in any training instance. We illustrate it in Comment 6.2. We prove the following.

TheOREM 6.1. Let $\Pi$ be a statistic in $\mathbf{Q L}_{\text {con }}$ such that every feature query $\pi$ in $\Pi$ is selective. The $V C$ dimension of $\operatorname{Lin}$ w.r.t. $\Pi$ is $d+1$, where $d$ is equal to the number of equivalence classes ${ }^{2}$ among the queries in $\Pi$.

Proof. We first show that the dimension is at least $d+1$. We need to construct an instance $I$ over $\mathbf{S}$ such that $I$ has $d+1$ entities, and for every labeling $\lambda$ of $I$ we can find a linear classifier that is consistent with $\lambda$. Let $q_{1}, \ldots, q_{d}$ be representatives of the $d$ equivalence classes of the feature queries in $\Pi$. We denote by $q_{0}$ the $\mathrm{CQ} Q(x) \leftarrow \eta_{\mathbf{S}}(x)$. Note that no two CQs in $q_{0}, \ldots, q_{d}$ are equivalent. Let $r_{0}, \ldots, r_{d}$ be an ordering of $q_{0}, \ldots, q_{d}$ in a topological order, so that if $r_{i} \subseteq r_{j}$ and $i \neq j$, then $i>j$. For $i=0, \ldots, d$, we define the instance $I_{i}$ to be a canonical instance of $r_{i}$, so that different $I_{i}$ have disjoint active domains. We add to each $I_{i}$ the entity $e_{i}$, which is the constant that corresponds to the head variable of $r_{i}$. The following hold for every $i=0, \ldots, d$.

$$
\begin{aligned}
& \text { 1. } e_{i} \in r_{i}\left(I_{i}\right) . \\
& \text { 2. } e_{j} \notin r_{i}\left(I_{j}\right) \text { if } i>j .
\end{aligned}
$$

\footnotetext{
${ }^{2}$ Query equivalence, as defined in Section 2.2.
} 
The reason for the second item is well known in the theory of CQs [11]: $e_{j} \in r_{i}\left(I_{j}\right)$ implies a homomorphism from $r_{i}$ to $I_{j}$ that maps the head to $e_{j}$, and since $I_{j}$ is the canonical instance of $r_{j}$ this homomorphism can also be viewed as a homomorphism $\mu_{i, j}$ from $r_{i}$ to $r_{j}$ that preserves the head variable. So, every assignment $\mu_{j, J}$ from $r_{j}$ to any instance $J$ can be composed with $\mu_{i, j}$ to form a homomorphism $\mu=$ $\mu_{j, J} \circ \mu_{i, j}$ from $r_{i}$ to $J$ so that $\mu(x)=\mu_{j, J}(x)$ for the head variable $x$, hence $r_{j} \subseteq r_{i}$, contradicting $i>j$.

We then define $I$ as $I_{0} \cup \cdots \cup I_{d}$. Observe that $I$ has $d+1$ entities, namely $e_{0}, \ldots, e_{d}$. Since each $r_{i}$ is connected and constant free, for every entity $e_{j}$ it is the case that $e_{j} \in r_{i}(I)$ if and only if $e_{j} \in r_{i}\left(I_{j}\right)$, since any homomorphism from $r_{i}$ to $I$ must be a homomorphism into a single $I_{k}$. From here on we continue similarly to Lemma 5.8. Specifically, let $\lambda$ be a labeling of $I$. We need to construct a linear classifier $h$ that is consistent with $\lambda$. The classifier $h$ is defined as

$$
\delta_{0}+\delta_{1}+\cdots+\delta_{d} \geq 0
$$

where and $\delta_{j}$ are defined as follows for $j=0, \ldots, m$ :

$$
\delta_{j} \stackrel{\text { def }}{=} \frac{1}{2}\left(\lambda\left(e_{j}\right)-\sum_{k=1}^{j-1} \delta_{k}\left(e_{j}\right)\right)\left(1+r_{j}^{I}\right)
$$

The proof that $h$ is consistent with $\lambda$ is as in the proof of Lemma 5.8. Observe that $\delta_{0}\left(e_{i}\right)$ is always 1 , since $r_{0}$ is the $\mathrm{CQ} q_{0}$ that selects all the entities. Hence, $\delta_{0}$ can be replaced by a constant, and so, it is indeed a classifier over $\Pi$.

We now need to prove that the VC dimension of Lin w.r.t. $\Pi$ is at most $d+1$. Assume, by way of contradiction, that $I$ is an instance over $\mathbf{S}$ such that $I$ has $d+2$ entities, and Lin shatters $I$ w.r.t. $\Pi$. To establish a contradiction, we will construct a sequence $S$ of $d+2$ vectors in $\{-1,1\}^{d}$ such that $S$ is shattered by $\operatorname{Lin}_{d}$. It is well known that no such set $S$ exists [33], and hence, we get a contradiction. So, in the remainder of this section we construct $S$.

Denote $\Pi$ as $\left(\pi_{1}, \ldots, \pi_{m}\right)$. If $i<j$ are such that $\pi_{i}$ is equivalent to $\pi_{j}$, then every $\Pi^{I}(e)$ has the same value in the $i$ th and $j$ th positions. Therefore, every linear function over $\Pi$ can be transformed into a linear function over $\Pi^{\prime}$, where $\Pi^{\prime}$ is obtained from $\Pi$ by removing $\pi_{j}$, while preserving the output values. Therefore, $I$ is shattered by $\Pi^{\prime}$ as well. We can continue to remove queries from $\Pi$ until we obtain a statistic $\Pi_{d}$ of arity $d$. Let $e_{1}, \ldots, e_{d+2}$ be the entities of $I$. Then we define $S$ to be the sequence $\Pi_{d}^{I}\left(e_{1}\right), \ldots, \Pi_{d}^{I}\left(e_{d+2}\right)$. By our assumption, the sequence $S$ is shattered by Lin.

Note that Theorem 6.1 implies that that computing the $\mathrm{VC}$ dimension considered class of CQs is not harder than computing equivalence. It is also not easier, since query equivalence can (quite straightforwardly) be reduced to computing the number of equivalence classes.

Comment 6.2. In Theorem 6.1, the assumption that $\Pi$ contains only selective feature queries is necessary. As an example, consider the statistic $\Pi$ that consists of only $q(x) \leftarrow$ $\eta_{\mathbf{S}}(x)$. Then $\Pi^{I}(e)=1$ for every instance $I$ and entity $e$ in $I$, and hence, no hypothesis class can shatter more than a single entity, and the $\mathrm{VC}$ dimension is 1 (rather than 2 ). Moreover, the assumption that $\Pi$ consists of connected CQs is also necessary. As an example, consider the statistic $\Pi$ that consists of the CQs $q(x) \leftarrow \eta_{\mathbf{S}}(x), R(x), P(y)$ and $q(x) \leftarrow \eta_{\mathbf{S}}(x), R(x), Q(z)$. Then two entities in $R$ will have the same features, and two entities outside of $R$ will have the same features; hence, the VC dimension is 2 (and not 3). We defer to future work the extension to the general case that avoids these assumptions.

\section{IDENTIFIABILITY WITH CQS}

We now investigate the identifiability problem in the case where feature queries are given as CQs. We first consider connected CQs, and prove two results: (1) if negation is allowed, then affine identifiability and linear identifiability are different properties (i.e., linear identifiability is strictly more restrictive than affine identifiability, cf. Fact 4.3), and (2), without negation, the two types of identifiability converge, and their identification is coNP-complete. In the second part, we generalize by lifting the restriction of connectivity, but there our characterization becomes more complicated, and our analysis requires the assumption of selectivity of every feature query.

\subsection{Connected CQs}

Recall Fact 4.3. The following shows that the opposite direction does not always hold for CQs with negation (even if we assume lack of self joins).

THEOREM 7.1. There is a statistic in $\mathrm{CQ}_{\mathrm{sjf}}$ that is affinely identifiable but not linearly identifiable.

Proof. We construct an entity schema $\mathbf{S}$ and a statistic $\Pi$ over $\mathbf{S}$. The schema $\mathbf{S}$ contains three unary relation symbols $\eta, R$ and $S$, with $\eta$ being $\eta_{\mathbf{S}}$. We then define $\Pi$ as $\left(\pi_{1}, \pi_{2}, \pi_{3}\right)$ where:

$$
\begin{aligned}
& \pi_{1}(x) \leftarrow \eta(x), R(x) \\
& \pi_{2}(x) \leftarrow \eta(x), \neg R(x) \\
& \pi_{3}(x) \leftarrow \eta(x), R(x), S(x)
\end{aligned}
$$

We will show that $\Pi$ is affinely identifiable but not linearly identifiable. We first prove that $\Pi$ is not linearly identifiable. Let $I$ be an instance over $\mathbf{S}$, and let $C_{1}$ and $C_{2}$ be the leftmost and middle columns of $\llbracket \Pi^{I} \rrbracket$, respectively. Then for every entity $e \in \eta_{\mathbf{S}}(I)$ we have $e \in \pi_{1}(x)$ if and only if $e \notin \pi_{2}(x)$. Therefore, it holds that $C_{1}=-C_{2}$, and so we have a linear dependence.

To show that $\Pi$ is affinely identifiable, we construct a specific instance $I$ with three entities $e_{1}, e_{2}$ and $e_{3}$, such that the $R$-facts of $I$ consist of $R\left(e_{1}\right)$ and $R\left(e_{3}\right)$, and the $S$-facts of $I$ consist of only $S\left(e_{3}\right)$. And so, the matrix $M$ is the following.

$$
M=\left[\begin{array}{lll}
+1 & -1 & -1 \\
-1 & +1 & -1 \\
+1 & -1 & +1
\end{array}\right]
$$

Let $C_{1}, C_{2}$ and $C_{3}$ be the columns of $M$ from left to right, respectively. Suppose that $a, b$ and $c$ are real numbers such that $a C_{1}+b C_{2}+c C_{3}=\mathbf{0}^{3}$ and $a+b+c=0$. We need to show that $a=b=c=0$. We have the following.

$$
\begin{aligned}
a-b-c & =0 \\
-a+b-c & =0 \\
a-b+c & =0
\end{aligned}
$$

From the first two equations we conclude that $c=0$. Therefore, $a=b$. And from $a+b+c=0$ we conclude that $a=b=c=0$, as claimed. 
In the remainder of this section we show that in the case of CQs (without negation), the two types of identifiability converge, and they amount to query equivalence. We begin with connected CQs. A statistic $\Pi$ is said to have redundancy if it contains two distinct feature queries that are equivalent.

THEOREM 7.2. Let $\Pi$ be a statistic in $\mathrm{CQ}_{\mathrm{con}}$. The following hold.

1. If $\Pi$ has redundancy, then $\Pi$ is not affinely identifiable (hence, also not linearly identifiable).

2. If $\Pi$ has no redundancy, then $\Pi$ is linearly identifiable (hence, also affinely identifiable).

Proof. Part 1 is straightforward: if $\Pi$ has redundancy then every matrix $\llbracket \Pi^{I} \rrbracket$ has two identical columns. We will prove Part 2.

Let $\Pi=\left(\pi_{1}, \ldots, \pi_{n}\right)$ be given. We assume that $\Pi$ has no redundancy. We will construct an instance $I$ over $\mathbf{S}$, and we will prove that the degree of $\llbracket \Pi^{I} \rrbracket$ is $n$ (hence, its columns are independent).

The instance $I$ is the union of $n+1$ instances $I_{0}, \ldots, I_{n}$, where every two have disjoint active domains. We construct the $I_{i}$ as follows. Without loss of generality, assume that no variable is shared among two or more $\pi_{i}$. For $i=1, \ldots, n$, we define $I_{i}$ to be a canonical instance of $\pi_{i}$. Hence, $I_{i}$ is obtained from $\pi_{i}$ by replacing every variable $x$ with a unique constant $c_{x}$. For each $i$, let $e_{i}$ be the tuple of constants that corresponds to the variable sequence in the head of $\pi_{i}$. We insert $e_{i}$ to the entity relation of $\eta_{I}$. Finally, we define $I_{0}$ to be an isomorphic copy of $I_{1} \cup \cdots \cup I_{n}$, where all the $e_{i}$ are replaced with a new entity $e_{0}$ (using fresh new constants), and we add $e_{0}$ to the entity relation of $I_{0}$.

It remains to prove that $\llbracket \Pi^{I} \rrbracket$ has $n$ independent rows. Let $r_{0}, \ldots, r_{n}$ be the rows that correspond to the entities $e_{0}, \ldots, e_{n}$. We will show that there are $n$ independent rows among $r_{0}, \ldots, r_{n}$. For that, it suffices to prove that the rows $r_{1}+r_{0}, \ldots, r_{n}+r_{0}$ are independent, and this is what we do. Observe that $r_{0}$ is a vector of $1 \mathrm{~s}$. Hence, $r_{i}+r_{0}$ is the vector $\left(v_{1}^{i}, \ldots, v_{n}^{i}\right)$ where:

$$
v_{j}^{i}= \begin{cases}2 & \text { if } e_{j} \in \pi_{i}(I) \\ 0 & \text { otherwise }\end{cases}
$$

For $i=1, \ldots, n$, let $r_{i}^{\prime}$ be the vector $r_{i}+r_{0}$. Suppose that $a_{1}, \ldots, a_{k}$ are real numbers satisfying

$$
\sum_{i=1}^{n} a_{i} r_{i}^{\prime}=0
$$

We need to show that $a_{i}=0$ for all $i=1, \ldots, n$. Consider the containment binary relation $\subseteq$ among the $\pi_{i}$ s. Since no two $\pi_{i}$ s are equivalent, the relation $\subseteq$ is a preorder. Without loss of generality (as no order was assumed between the $\pi_{i}$ ), assume that $\pi_{n}$ is a minimal element in the $\subseteq$ relationship. Then there is no $\pi_{i}$ such that $i<n$ and $\pi_{i} \subseteq \pi_{n}$. If for some $i<n$ we have $e_{i} \in \pi_{n}(I)$, then we have $\pi_{i} \subseteq \pi_{n}$ due to the fact that all $\pi_{j}$ s are connected, and that our construction is based on canonical instances. Therefore, for $i<n$ we have $e_{i} \notin \pi_{n}(I)$. In particular, for $i<n$ we have that $r_{i}^{\prime}$ contains 0 in its $n$th component. And of course, $r_{n}^{\prime}$ contains 2 in its $n$th component. We then conclude that $a_{n}=0$.

It then follows that $\sum_{i=1}^{n-1} a_{i} r_{i}^{\prime}=0$, and we can continue iteratively and prove that each $a_{i}$ is zero. This concludes the proof.
By applying the NP-completeness of CQ containment and equivalence [11] we get that identifiability is coNP-complete in the case of connected CQs.

COROLlary 7.3. Both linear and affine $\mathrm{CQ}_{\text {con-identifiability }}$ are coNP-complete.

Next, we show that the assumptions of lack of constants (that we make throughout the paper) and connectivity are necessary for the correctness of Theorem 7.2. We begin with the lack of constants.

EXAMPLE 7.4. Let $\mathbf{S}$ be an entity schema with $\eta_{\mathbf{S}}=\eta$ and $\Pi=\left(\pi_{1}, \pi_{2}, \pi_{3}, \pi_{4}\right)$ be the statistic defined as follows.

$$
\begin{aligned}
& \pi_{1}(x) \leftarrow \eta(x), S(x, \text { 'a') } \\
& \pi_{2}(x) \leftarrow \eta(x), S(x, \text { 'a'), } S(x, y), T(y, z) \\
& \pi_{3}(x) \leftarrow \eta(x), W(x, z), T(\text { ' } \mathrm{a} \text { ' }, z) \\
& \pi_{4}(x) \leftarrow \eta(x), V(x, z), T(\text { ' } \mathrm{a} \text { ' }, z)^{2}
\end{aligned}
$$

Note that these feature queries are connected, but not in $\mathrm{CQ}_{\text {con }}$, since we do not allow constants in $\mathrm{CQ}_{\text {con }}$. No two feature queries in $\Pi$ are equivalent, but we will show that $\Pi$ is not affinely identifiable. Let $I$ be an instance over $\mathbf{S}$. We consider two cases.

- Case 1: No entity satisfies $\pi_{3}$ and $\pi_{4}$. Then in this case, the matrix $\llbracket \Pi^{I} \rrbracket$ has two identical columns (namely, the third and fourth).

- Case 2: At least one entity satisfies $\pi_{3}$ or $\pi_{4}$. Then in this case, we know that $I$ contains $T$ (' $\mathrm{a}$ ', $z$ ) for some $z$, and hence, every entity $x$ satisfies $\pi_{1}$ if and only if it satisfies $\pi_{2}$. Therefore, $\llbracket \Pi^{I} \rrbracket$ again contains two identical columns (namely the first and the second).

We conclude that $\llbracket \Pi^{I} \rrbracket$ contains two identical columns, and therefore, has an affine (and linear) dependency.

The next example shows that Theorem 7.2 is not necessarily true if we avoid the assumption of connectivity.

EXAMPLE 7.5. Let $\mathbf{S}$ be an entity schema with $\eta_{\mathbf{S}}=\eta$ and the relation symbols $S / 1, T / 1$ and $W / 1$, and $\Pi=$ $\left(\pi_{1}, \pi_{2}, \pi_{3}, \pi_{4}\right)$ be the statistic defined as follows.

$$
\begin{aligned}
& \pi_{1}(x) \leftarrow S(x) \\
& \pi_{2}(x) \leftarrow S(x), T(y) \\
& \pi_{3}(x) \leftarrow W(x), T(x) \\
& \pi_{4}(x) \leftarrow V(x), T(x)
\end{aligned}
$$

Observe that no two features in $\Pi$ are equivalent. Nevertheless, $\Pi$ is not affinely identifiable, and this can be shown using the same arguments of Example 7.4.

\subsection{Disconnected CQs}

We now extend Theorem 7.2 to handle disconnected CQs. For that, we need some notation. Let $\pi$ be a CQ feature query. We denote $\pi$ as

$$
\pi(x) \leftarrow \exists \mathbf{y}, \mathbf{z}[\phi(\mathbf{y}) \wedge \psi(\mathbf{z})]
$$

where $\phi(\mathbf{y})$ is the conjunction of atoms that consists of the connected component of $x$, the vectors $\mathbf{y}$ and $\mathbf{z}$ are disjoint, and $\psi(\mathbf{z})$ is the conjunction of the remaining atoms. We denote by $\pi^{c}$ and $\pi^{d}$ the restrictions of $\pi$ to $\phi$ and $\psi$, respectively. Hence, $\pi^{c}$ is a feature query, and $\pi^{d}$ is a Boolean 
query. Finally, we use $\pi^{b}$ to denote the Boolean version of $\pi$, namely "there exists $x$ such that $\pi(x) . "$ More formally, we have defined the following three CQs.

$$
\begin{aligned}
\pi^{c}(x) & \leftarrow \exists \mathbf{y}[\phi(\mathbf{y})] \\
\pi^{d}() & \leftarrow \exists \mathbf{z}[\psi(\mathbf{z})] \\
\pi^{b}() & \leftarrow \exists x, \mathbf{y}, \mathbf{z}[\phi(\mathbf{y}) \wedge \psi(\mathbf{z})]
\end{aligned}
$$

DeFinition 7.6. Let $\Pi=\left(\pi_{1}, \ldots, \pi_{n}\right)$ be a statistic in CQ. We say that $\Pi$ is weakly redundant if one of the following holds.

1. There are two or more distinct pairs of equivalent queries among $\pi_{1}^{c}, \ldots, \pi_{n}^{c}$.

2. $\pi_{i}^{c}$ and $\pi_{j}^{c}$ are equivalent for some $1 \leq i<j \leq n$ and both of the following hold.

- $\pi_{i}^{d}$ is logically entailed by the conjunction of the $\pi_{l}^{b}$ for $l \neq i$.

- $\pi_{j}^{d}$ is logically entailed by the conjunction of the $\pi_{l}^{b}$ for $l \neq j$.

We can now state the main theorem for this section.

THEOREM 7.7. Let $\mathbf{S}$ be an entity schema, and let $\Pi$ be a statistic in CQ such that $\pi_{i}^{c}$ is selective for all $\pi$ in $\Pi$.

1. If $\Pi$ has weak redundancy, then $\Pi$ is not affinely identifiable (hence, also not linearly identifiable).

2. If $\Pi$ has no weak redundancy, then $\Pi$ is linearly identifiable (hence, also affinely identifiable).

The proof of Theorem 7.7 is in the appendix. We conclude the following.

COROLlary 7.8. Under the assumption that every feature query $\pi$ is such that $\pi^{c}$ is selective, both linear and affine CQ-identifiability are coNP-complete.

Let us now remark on the assumption made in Theorem 7.7 that every $\pi^{c}$ is selective. Consider the statistic $\Pi=\left(\pi_{1}, \pi_{2}\right)$ that is defined as follows.

$$
\pi_{1}(x) \leftarrow \eta_{\mathbf{S}}(x) \quad \pi_{2}(x) \leftarrow \eta_{\mathbf{S}}(x), T(y)
$$

It is easy to see that $\Pi$ is not linearly identifiable, since in every $\llbracket \Pi^{I} \rrbracket$ each column will be a either $\mathbf{1}^{2}$ or $-\mathbf{1}^{2}$. However, $\Pi$ is affinely identifiable, since the instance $I$ with two entities and an empty $T$ satisfies

$$
\llbracket \Pi^{I} \rrbracket=\left[\begin{array}{ll}
+1 & -1 \\
+1 & -1
\end{array}\right]
$$

which is column affinely independent. Hence, a more careful analysis should be made in the case where non-selective feature queries are allowed.

\section{RELATED WORK}

The task of feature engineering has been widely studied for decades [10,20-23]. Our approach borrows heavily from the feature-engineering process identified in Guyon's seminal book [21] and those we have observed in practice. Feature engineering has received some attention from the database community $[2,3,32,37]$. That work has made algorithmic or tooling contributions to better support feature engineering, but they do not answer the fundamental questions for which our framework is designed.

Frameworks and query languages that fuse logic with probabilistic semantics, to simplify the design of machine-learning models, have been proposed and developed in past decades. Examples of these include Probabilistic Relation Models pioneered by Koller and Friedman [18], PRISM [30], BLOG [28], Markov Logic Networks [29], and the recent ProbabilisticProgramming Datalog [7]. However, these approaches focus on orthogonal formal questions: the semantics of the models and the complexity of the associated inference tasks. In contrast, we consider the interplay of the logical rules and learning properties. In particular, to the best of our knowledge this work is the first to consider separability, identifiability, and $\mathrm{VC}$ dimensionality in machine-learning models that are defined over database queries. Another related approach is Probabilistic Soft Logic (PSL) where Getoor et al. [6] modify the way in which the objective function is constructed for the task to preserve convexity, which is desirable for the sake of efficiency. All of our classifier models are convex, but this does not obviate the need to understand identifiability and VC dimension, which are finer information about the model.

Our formal framework draws inspiration from previous approaches to combining logical reasoning to probabilistic reasoning, which is a classical topic $[5,15]$, but is distinct in its goal. Our focus is on the process of feature engineering, which motivates the computational problems that occur during this loop. On the machine-learning side, we build on the conditions for identifiability described in Wainwright and Jordan's survey [35].

There has been a lot of work in the Machine Learning community on learnability aspects of First Order formulas. For instance, Arias and Khardon [4] have considered such aspects (including VC dimension) in the context of Horn clauses, where they establish bounds that are based on syntactic properties of the clauses (e.g., number of variables, literals, clauses, etc.). Such a setup is quite different from ours, since there the goal is to classify a whole interpretation (database) based on a single formula (to be learned), while we consider classification of entities within a single database and focus on feature engineering rather than the engineering of the classifier. More technically, our focus here is not on the syntactic properties of queries, but rather on the relationship among different feature queries. Finally, while the bounds of Arias and Khardon [4] are based on syntactic properties of Horn clauses, ours are based on equivalences. In particular, our proof techniques are quite different and draw the connection to traditional database theory.

\section{CONCLUDING REMARKS}

We have developed a framework for feature engineering towards programming machine-learning solutions, while focusing on the important task of classification. Our framework is based on simple additions to the relational data and query model, where an entity schema allows to represent entities along with their associated information, and where feature engineering is the task of designing a statistic when given a training instance over the entity schema. This framework enables us to formalize realistic problems in a manner that allows for nontrivial analysis and, consequently, insights and solutions. In particular, we have formalized three important computational problems within our framework: separability, identifiability, and VC dimensionality. These problems are 
parameterized by the hypothesis class in use and the query language deployed for feature extraction.

We have presented insights and complexity results on the three problems, while focusing on features definable by conjunctive queries and on linear hypotheses. We have drawn connections between these problems and those of query containment and equivalence. These connections have several important consequences. First, there is a tight relationship between the computational complexity of our problems and that of query containment: it is both necessary and sufficient to solve CQ containment in order to solve our problems. Second, the fact that identifiability "comes for free" (up to redundancy) gives a formal indication of the suitability of CQs as a language for feature engineering. It also motivates the challenge of finding other natural query languages that are likewise suitable.

We view the analysis we have done in this paper as providing preliminary results and a baseline for future research within our proposed framework and extensions thereof. We conclude this paper with a number of proposed directions and extensions for such future research. These fall in two main categories: logical analysis and statistical questions.

\section{Logical Analysis}

Further expressiveness. We have focused on the simple class of conjunctive queries for defining statistics, and on the classifier class of linear hypotheses. An obvious future direction would be to consider more expressive classes, such as queries with additional logical operators (and in particular deepen the exploration of non-monotonic features), and aggregate functions.

Schema constraints. Some of the tasks we have considered in this paper would be greatly impacted if we allowed for schema constraints. In particular, in the identifiability problem column independence would need to be realized by an instance that satisfies the constraints, and not by any instance of the signature (as our proofs assumed). The problem of VC dimensionality would be similarly impacted. We view this direction as an important opportunity of incorporating the database's rich modeling of data into the task of feature engineering.

Text analysis. An area where machine-learning classification is crucial for even simple tasks is that of text analysis, and in particular when the text is natural language from open domains such as Web and social media [32]. Consequently, we belive that a direction of a high potential impact is that of applying our framework to domains that allows queries over text, such as the document spanners of Fagin et al. $[16,17]$ that construct and manipulate relations over text spans (intervals) using extractors (e.g., regular expressions). In particular, the computational challenges will involve queries with both relational and textual operations.

\section{Statistical Questions}

Generalized learning tasks. Our features in this work were all Boolean $( \pm 1)$, and it is desirable to study the natu- ral extension of the framework to numerical features, where numbers are either directly copied from the database or indirectly computed via queries. Moreover, our framework can be easily generalized to other prediction tasks, such as multilabel classification (e.g., predict the age group of a person) and numerical regression (e.g., predict the actual age of the person). It is important to understand how the challenges we considered are affected by such generalizations.

Separability relaxation. The separability problem, as defined in this paper, can be extended by allowing for an $a p$ proximate agreement with the training examples (e.g., the hypothesis $h$ should agree with the labeling $\lambda$ on at least $(1-\epsilon)$ of the entities, or at most $k$ entities should be misclassified). This is a practical and crucial relaxation in practical scenarios. For one, the training data may be noisy. Moreover, our hypothesis class may be too simple to precisely cover the examples, but can do so with only a small error.

Model complexity. In parallel to extending the expressiveness of queries, it is of high importance to find the proper restrictions on the engineered statistics (a.k.a. regularization), in order to reduce the model complexity and, consequently, reduce the risk of overfitting to the training samples (and, orthogonally, gain more efficient machine-leaning solutions). The common regularization limits the length of the statistic; in our framework, we can consider restrictions on the feature queries (e.g., size, depth, number of constants/variables, etc.). The ultimate goal is to find settings that properly balance between overfitting, underfitting, inference (classification) complexity and learning (training) complexity.

The vast literature on machine learning gives rise to many more directions for our framework to extend, such as notions of capacity beyond VC dimension (e.g., Rademacher and Gaussian complexities [8]) and the implications of the "transductive" learning environments, where we know to begin with what entities we will need to predict upon [19]. We believe that our framework can contribute to many of these directions the important angle of data and query modelling.

\section{Acknowledgments}

The authors are grateful to Stephen Bach and Alex Ratner for valuable suggestions on this work and on its presentation in this paper. Benny Kimelfeld is a Taub Fellow, supported by the Taub Foundation. His research is also supported by the Israeli Science Foundation, Grants 1295/15 and 1308/15. The research of Christopher Ré is supported by DARPA's projects XDATA (FA8750-12-2-0335), DEFT (FA8750-132-0039), MEMEX, and SIMPLEX. His research is also supported by NSF Career Award IIS-1353606, ONR Awards N000141210041 and N000141310129, NIH Grant U54EB02005 (awarded by NIBIB through funds provided by the trans-NIH BD2K initiative), the Sloan Research Fellowship, the Moore Foundation, American Family Insurance, Google, and Toshiba. Any opinions, findings, and conclusions or recommendations expressed in this material are those of the authors and do not necessarily reflect the views of DARPA, AFRL, NSF, ONR, NIH, or the U.S. government. 


\section{REFERENCES}

[1] SAS Report on Analytics. sas.com/reg/wp/corp/23876.

[2] M. Anderson, D. Antenucci, V. Bittorf, M. Burgess, M. Cafarella, A. Kumar, F. Niu, Y. Park, C. Ré, and C. Zhang. Brainwash: A Data System for Feature Engineering. In $C I D R, 2013$.

[3] M. R. Anderson, M. J. Cafarella, Y. Jiang, G. Wang, and B. Zhang. An integrated development environment for faster feature engineering. PVLDB, 7(13):1657-1660, 2014.

[4] M. Arias and R. Khardon. Complexity parameters for first order classes. Machine Learning, 64(1-3):121-144, 2006.

[5] F. Bacchus, A. J. Grove, J. Y. Halpern, and D. Koller. From statistical knowledge bases to degrees of belief. CoRR, cs.AI/0307056, 2003.

[6] S. H. Bach, M. Broecheler, B. Huang, and L. Getoor. Hinge-loss markov random fields and probabilistic soft logic. CoRR, abs/1505.04406, 2015.

[7] V. Bárány, B. ten Cate, B. Kimelfeld, D. Olteanu, and Z. Vagena. Declarative probabilistic programming with datalog. In ICDT, volume 48 of LIPICs, pages 7:1-7:19. Schloss Dagstuhl - Leibniz-Zentrum fuer Informatik, 2016.

[8] P. L. Bartlett and S. Mendelson. Rademacher and gaussian complexities: Risk bounds and structural results. In COLT, pages 224-240, 2001.

[9] Y. Bengio and A. C. Courville. Deep learning of representations. In Handbook on Neural Information Processing, volume 49 of Intelligent Systems Reference Library, pages 1-28. Springer, 2013.

[10] D. E. Boyce. Optimal Subset Selection: Multiple Regression, Interdependence, and Optimal Network Algorithms . Springer-Verlag, 1974.

[11] A. K. Chandra and P. M. Merlin. Optimal implementation of conjunctive queries in relational data bases. In J. E. Hopcroft, E. P. Friedman, and M. A. Harrison, editors, STOC, pages 77-90. ACM, 1977 .

[12] C. Chekuri and A. Rajaraman. Conjunctive query containment revisited. Theor. Comput. Sci., 239(2):211-229, 2000.

[13] S. Cohen and Y. Y. Weiss. Learning tree patterns from example graphs. In ICDT, pages 127-143, 2015.

[14] C. Dwork, M. Hardt, T. Pitassi, O. Reingold, and R. S. Zemel. Fairness through awareness. In ITCS, pages 214-226. ACM, 2012.

[15] R. Fagin, J. Y. Halpern, and N. Megiddo. A logic for reasoning about probabilities. Inf. Comput., 87(1/2):78-128, 1990.

[16] R. Fagin, B. Kimelfeld, F. Reiss, and S. Vansummeren. Spanners: a formal framework for information extraction. In PODS, pages 37-48, 2013.

[17] R. Fagin, B. Kimelfeld, F. Reiss, and S. Vansummeren. Document spanners: A formal approach to information extraction. J. ACM, 62(2):12, 2015.

[18] N. Friedman, L. Getoor, D. Koller, and A. Pfeffer. Learning probabilistic relational models. In IJCAI, pages 1300-1309, 1999.

[19] A. Gammerman, K. S. Azoury, and V. Vapnik.
Learning by transduction. In $U A I$, pages 148-155. Morgan Kaufmann, 1998.

[20] I. Guyon and A. Elisseeff. An introduction to variable and feature selection. Journal of Machine Learning Research, 3:1157-1182, 2003.

[21] I. Guyon, S. Gunn, M. Nikravesh, and L. A. Zadeh. Feature Extraction: Foundations and Applications (Studies in Fuzziness and Soft Computing). Springer-Verlag New York, Inc., Secaucus, NJ, USA, 2006.

[22] T. Hastie, R. Tibshirani, and J. Friedman. The Elements of Statistical Learning: Data mining, inference, and prediction. Springer, 2001.

[23] G. H. John, R. Kohavi, and K. Pfleger. Irrelevant features and the subset selection problem. In Machine Learning: Proceedings of the Eleventh International Conference, pages 121-129, 1994.

[24] S. Kandel, A. Paepcke, J. M. Hellerstein, and J. Heer. Enterprise data analysis and visualization: An interview study. IEEE Trans. Vis. Comput. Graph., 18(12):2917-2926, 2012.

[25] B. Kimelfeld and P. G. Kolaitis. The complexity of mining maximal frequent subgraphs. ACM Trans. Database Syst., 39(4):32:1-32:33, 2014.

[26] Y. LeCun, Y. Bengio, and G. Hinton. Deep learning. Nature, 521(7553):436-444, 2015.

[27] E. L. Lehmann and G. Casella. Theory of point estimation, volume 31. Springer, 1998.

[28] B. Milch, B. Marthi, S. J. Russell, D. Sontag, D. L. Ong, and A. Kolobov. Blog: Probabilistic models with unknown objects. In IJCAI, pages 1352-1359, 2005.

[29] M. Richardson and P. Domingos. Markov logic networks. Mach. Learn., 62(1-2):107-136, 2006.

[30] T. Sato and Y. Kameya. PRISM: A language for symbolic-statistical modeling. In IJCAI, pages 1330-1339, 1997.

[31] S. Shalev-Shwartz and S. Ben-David. Understanding Machine Learning: From Theory to Algorithms. Cambridge University Press, 2014.

[32] J. Shin, S. Wu, F. Wang, C. D. Sa, C. Zhang, and C. Ré. Incremental knowledge base construction using DeepDive. PVLDB, 8(11):1310-1321, 2015.

[33] V. Vapnik. An overview of statistical learning theory. IEEE Transactions on Neural Networks, 10(5):988-999, 1999.

[34] V. N. Vapnik and A. Y. Chervonenkis. On the uniform convergence of relative frequencies of events to their probabilities. Theory of Probability and its Applications, 16(2):264-280, 1971.

[35] M. J. Wainwright and M. I. Jordan. Graphical models, exponential families, and variational inference. Foundations and Trends in Machine Learning, 1(1-2):1-305, 2008.

[36] R. S. Zemel, Y. Wu, K. Swersky, T. Pitassi, and C. Dwork. Learning fair representations. In ICML, volume 28 of JMLR Proceedings, pages 325-333. JMLR.org, 2013.

[37] C. Zhang, A. Kumar, and C. RÃl'. Materialization optimizations for feature selection workloads. In SIGMOD Conference, pages 265-276, 2014. 


\section{APPENDIX}

\section{A. ADDITIONAL PROOFS}

\section{A.1 Proof of Theorem 5.2}

We now prove Theorem 5.2. The following lemma states membership in $\Sigma_{2}^{\mathrm{P}}$.

Lemma A.1. (Lin, $\mathrm{CQ}_{\mathrm{sjf}}$ )-separability is in $\Sigma_{2}^{\mathrm{P}}$.

Proof. Given an entity schema $\mathbf{S}$ and a training instance $(I, \lambda)$ over $\mathbf{S}$, an evidence for separability can be a statistic $\Pi=\left(\pi_{1}, \ldots, \pi_{n}\right)$ in $\mathrm{CQ}_{\mathrm{sjf}}$ where $n=\left|\eta_{\mathbf{S}}^{I}\right|$, such that there is a linear separation between the negative $\Pi(e)$ and the positive $\Pi(e)$. Proving every +1 in $\Pi(e)$ amounts to showing a homomorphism from the corresponding $\pi_{i}$ to $I$, and so is refuting every -1 . Observe that each $\pi_{i}$ is of linear size in $\mathbf{S}$, since $\pi_{i}$ does not have self joins. Determining whether there is a linear separator can be done in polynomial time by translation to Linear Programming (with both strict and non-strict inequalities). So, we get the following $\Sigma_{2}^{\mathrm{P}}$ representation of the problem: There exist a statistic $\Pi$ and vectors $v_{1}, \ldots, v_{m} \in\{-1,1\}^{n}$ corresponding to $\Pi\left(e_{1}\right), \ldots, \Pi\left(e_{n}\right)$, a linear separation between the positive and negative points, and a suitable homomorphism for every 1 in the $v_{j}$, such that for every -1 in the $v_{j}$ and for every function $\mu$ from the corresponding $\pi_{j}$ to $I$, the function $\mu$ is not a suitable homomorphism. This shows membership in $\Sigma_{2}^{\mathrm{P}}$, as claimed.

We now prove $\Sigma_{2}^{\mathrm{P}}$-hardness. Our proof is by a reduction from the $\Pi_{2}^{\mathrm{P}}$-complete problem $\mathrm{QCNF}_{2}$ to the complement of separability. The problem $\mathrm{QCNF}_{2}$ is defined as follows. Given a 3-CNF formula $\varphi(\mathbf{x}, \mathbf{y})$, determine whether it is the case that for every truth assignment to $\mathrm{x}$ there exists a truth assignment to $\mathbf{y}$ such that the two assignments satisfy $\varphi$.

Reduction. Let $\varphi(\mathbf{x}, \mathbf{y})$ be given. We will construct an entity schema $\mathbf{S}$ and a training instance $(I, \lambda)$ with two entities $a$ and $b$, such that $\lambda(a)=1$ and $\lambda(b)=-1$, and show that $a$ can be separated from $b$ by a CQ in $C_{\mathrm{sjf}}$ if and only if there exists an assignment to $\mathbf{x}$ such that $\varphi(\mathbf{x}, \mathbf{y})$ is violated by any assignment to $\mathbf{y}$.

We use the following notation. Recall that the input is $\varphi(\mathbf{x}, \mathbf{y})$. We assume $\mathbf{x}=\left(x_{1}, \ldots, x_{k}\right)$ and $\mathbf{y}=\left(y_{1}, \ldots, y_{l}\right)$. We denote $\varphi$ as $c_{1} \wedge \cdots \wedge c_{m}$, where each $c_{i}$ is a disjunction of three atomic formulas over $(\mathbf{x}, \mathbf{y})$. Each occurence of an atomic formula $\alpha_{j}$ in a clause $c_{i}=\alpha_{1} \vee \alpha_{2} \vee \alpha_{3}$ is associated with a unique position that we denote by $p=(i, j)$. We denote the atomic formula at position $p$ by $\alpha_{p}$ and the variable in $\alpha_{p}$ as $v_{p}$. Two positions $p$ and $p^{\prime}$ are in agreement if $\alpha_{p}=\alpha_{p^{\prime}}$.

We construct the entity schema $\mathbf{S}$ with the following relation symbols.

- $\eta / 1$ is the entity relation symbol.

- $\gamma / 2$; a fact $\gamma(e, r)$ states that the $r$ is a branch of the entity $e$.

- $C_{i} / 4$ for each clause $c_{i}$; a fact $C_{i}\left(r, g_{1}, g_{2}, g_{3}\right)$ denotes an assignment $\left(g_{1}, g_{2}, g_{3}\right)$ to the three variables of $c_{i}$, respectively, and this assignment is associated with the branch $r$.

- $X^{x, d} / 1$ for each variable $x$ in $\mathbf{x}$ and $d \in\{0,1\}$; the fact $X^{x, d}(g)$ denotes that $g$ represents an assignment of $d$ to $x$.
We now construct the instance $I$ over $\mathbf{S}$, as follows. There are precisely two entities $a$ and $b$ in $I$ (and those occur in the relation $\eta^{I}$ ). We construct instances $I_{a}$ and $I_{b}$ over $\mathbf{S}$. We further construct an instance $I_{b}^{p, p^{\prime}}$ for every pair $p$ and $p^{\prime}$ of positions in agreement. The active domains of these instances are pairwise disjoint, except that $I_{b}$ and $I_{b}^{p, p^{\prime}}$ share the entity $b$. Finally, we define $I$ as the union of $I_{a}, I_{b}$ and all of the $I_{b}^{p, p^{\prime}}$.

To construct the instance $I_{a}$, we define a fresh constant $g_{a}^{v, d}$ for every variable $v$ in $(\mathbf{x}, \mathbf{y})$ and $d \in\{0,1\}$. We then add to $I_{a}$ the following facts:

- $\eta(a)$.

- $\gamma(a, a)$.

- $C_{i}\left(a, g_{a}^{v_{1}, d_{1}}, g_{a}^{v_{2}, d_{2}}, g_{a}^{v_{3}, d_{3}}\right)$ for the variables $v_{1}, v_{2}$ and $v_{3}$ at positions $(i, 1),(i, 2)$ and $(i, 3)$, respectively, and for all $d_{1}, d_{2}$ and $d_{3}$ in $\{0,1\}$. In particular, $I_{a}$ contains 8 facts of $C_{i}$.

- $X^{x, d}\left(g_{a}^{x, d}\right)$ for all $x$ in $\mathbf{x}$ and $d \in\{0,1\}$.

The instance $I_{b}$ is constructed in the same way as $I_{a}$, except for the following differences.

- Every occurence of $a$ becomes $b$ (hence, $I_{a}$ and $I_{b}$ are domain disjoint).

- We do not add the fact $C_{i}\left(b, g_{b}^{v_{1}, d_{1}}, g_{b}^{v_{2}, d_{2}}, g_{b}^{v_{3}, d_{3}}\right)$ if it represents an assignment that violates the clause $c_{i}$. In particular, $I_{b}$ contains 7 facts of $C_{i}$.

Similarly, the instance $I_{b}^{p, p^{\prime}}$ is constructed in the same way as $I_{a}$, except for the following differences.

- Every occurence of $a$ becomes $b$.

- The fact $\gamma(a, a)$ is replaced by $\gamma\left(b,\left\langle p, p^{\prime}\right\rangle\right)$.

- Every occurrence of a constant $g_{a}^{v, d}$ becomes $g_{b, p, p^{\prime}}^{v, d}$, which is a fresh new constant.

- If $p=(i, j)$, then we do not add any fact of the form $C_{i}\left(b, g_{b, p, p^{\prime}}^{v_{1}, d_{1}}, g_{b, p, p^{\prime}}^{v_{2}, d_{2}}, g_{b, p, p^{\prime}}^{v_{3}, d_{3}}\right)$ with $d_{j}=1$.

- If $p^{\prime}=\left(i^{\prime}, j^{\prime}\right)$, then we do not add any fact of the form $C_{i}\left(b, g_{b, p, p^{\prime}}^{v_{1}, d_{1}}, g_{b, p, p^{\prime}}^{v_{2}, d_{2}}, g_{b, p, p^{\prime}}^{v_{3}, d_{3}}\right)$ with $d_{j^{\prime}}=0$.

We have defined $\mathbf{S}$ and $I$. As said above, we define $\lambda$ by $\lambda(a)=1$ and $\lambda(b)=-1$. This completes the description of the reduction.

Correctness. We need to show that there is a CQ in $\mathrm{CQ}_{\mathrm{sjf}}$ that separates $a$ from $b$ if and only if $\varphi(\mathbf{x}, \mathbf{y})$ is a "no" instance. Hence, we prove the following lemmas.

Lemma A.2. Suppose that there is an assignment for $\mathbf{x}$ such that no assignment for $\mathbf{y}$ satisfies $\varphi$. Then there is a $C Q \pi$ in $\mathrm{CQ}_{\mathrm{sjf}}$ such that $\pi$ separates a from $b$.

Proof. Let $\tau: \mathbf{x} \rightarrow\{0,1\}$ be an assignment for $\mathbf{x}$ as defined in the lemma. We denote $\tau\left(x_{i}\right)$ by $d_{i}$. We define the following $\mathrm{CQ} \pi$, using $\mathbf{x}$ and $\mathbf{y}$ as variables.

$$
\begin{aligned}
\pi_{0}(z) \leftarrow & \gamma(z, r), X^{x_{1}, d_{1}}\left(x_{1}\right), \ldots, X^{x_{k}, d_{k}}\left(x_{k}\right), \\
& C_{1}\left(r, v_{(1,1)}, v_{(1,2)}, v_{(1,3)}\right), \ldots, \\
& C_{m}\left(r, v_{(m, 1)}, v_{(m, 2)}, v_{(m, 3)}\right)
\end{aligned}
$$

Note that $v_{(i, j)}$ is the variable at position $(i, j)$, and so, different $C_{i}$ may share variables. The CQ $\pi_{0}(z)$ states that there is an assignment to $(\mathbf{x}, \mathbf{y})$ that agrees with $\tau$ on $\mathbf{x}$ and 
can be matched in the $C_{i}$ with the entity $z$. For $z=r=a$, the corresponding facts in $C_{i}$ match against every possible assignment, and in particular, such an assignment to $(\mathbf{x}, \mathbf{y})$ necessarily exists. We conclude that $a \in \pi_{0}(I)$.

We now need to show that $b \notin \pi_{0}(I)$. Suppose, by way of contradiction, that $\mu$ is a homomorphism from $\pi_{0}$ to $I$, such that $\mu(z)=b$. From our construction of $I$ and the fact that $Q$ is connected it follows that $\mu$ is a homomorphism to either $I_{b}$ or one of the $I_{b}^{p, p^{\prime}}$. Now, $\mu$ cannot be a homomorphism into $I_{b}^{p, p^{\prime}}$ since $\mu$ maps the same variable to every pair of attributes in agreement, and our construction of $I_{b}^{p, p^{\prime}}$ guarantees that $p$ and $p^{\prime}$ do not have the same value in $I_{b}^{p, p^{\prime}}$. It thus follows that $\mu$ is a homomorphism into $I_{b}$. But then, $\mu$ represents a legal assignment that agrees with $\tau$ (due to $\left.X^{x_{1}, d_{1}}\left(x_{1}\right), \ldots, X^{x_{k}, d_{k}}\left(x_{k}\right)\right)$. Moreover, the construction of $I_{b}$ implies that this legal assignment is actually a satisfying assignment of $\varphi$. We therefore get a contradiction to the assumption of the lemma.

LEMmA A.3. Suppose that for each assignment for $\mathbf{x}$ there is an assignment for $\mathbf{y}$ that satisfies $\varphi$. Then no $C Q \pi$ in $\mathrm{CQ}_{\mathrm{sjf}}$ separates a from $b$.

Proof. Let $\pi(z)$ be a feature query in $C_{\mathrm{sjf}}$. We need to show that $a \in \pi(I)$ if and only if $b \in \pi(I)$. If $\pi$ is not connected, then its part that is disconnected from $z$ must be satisfied, unless neither $a$ nor $b$ are in $\pi(I)$ and we are done. So the question is about whether or not there is a homomorphism from the part that is connected to $z$. Therefore, we will assume that $\pi$ is connected to begin with.

The "if" direction. Suppose that $\mu$ is a homomorphism from $\pi$ to $I$ such that $b \in \pi(I)$. Then, due to the fact that $\pi$ is connected and has no self joins, we get that $\pi$ is necessarily a homomorphism into either $I_{b}$ or some $I_{b}^{p, p^{\prime}}$. From our construction of these instances it follows that there is a homomorphism from $I_{b}$ to $I_{a}$ and from each $I_{b}^{p, p^{\prime}}$ to $I_{a}$. Hence, there is a homomorphism $\mu^{\prime}$ from $\pi$ to $I_{a}$. And since such a homomorphism must map the head variable $z$ into $a$ we get that $a \in \pi(I)$.

The "only if" direction. Suppose that $\mu$ is a homomorphism from $\pi$ to $I$ such that $a \in \pi(I)$. Again, $\pi$ is necessarily a homomorphism into $I_{a}$. We can view $\mu$ as a mapping of occurrences of variables in $\varphi(\mathbf{x}, \mathbf{y})$ into values. For example, if $\pi$ contains $C_{i}(r, x, y, z)$ and $\pi$ maps $x$ to $g_{a}^{v_{j}, d_{j}}$, then we get the assignment $v_{j} \rightarrow d_{j}$. Nevertheless, this assignment need not be consistent (i.e., $v_{j}$ may be mapped to 1 in one place, and to 0 in another place). If that is the case, then let $p$ and $p^{\prime}$ be positions that give rise to inconsistency. Then, from the construction of $I_{b}^{p, p^{\prime}}$ we can change $\mu$ to be a homomorphism to either $I_{b}^{p, p^{\prime}}$ or $I_{b}^{p^{\prime}, p}$ (depending on whether the value at $p$ is 0 or 1 , respectively).

We now assume that $\mu$ represents a consistent assignment. We can replace every pair of variables in $\pi$ to a new common variable whenever these are in positions that correspond to the same variable of $\varphi$. (Note that $\pi$ cannot use the same variable for two different variables of $\varphi$, since $I_{a}$ has different values in the corresponding positions.) We can further assume that the variables have the same names as used in the feature query $\pi_{0}$ defined in the proof of Lemma A.2. And we can add to $\pi$ all the $C_{i}$ atoms of $\pi_{0}$. Every atom $X^{x_{i}, d_{i}}(w)$ in $\pi$ needs to be connected to some $C_{i}$ atom (as $\pi$ is connected), and since $\mu$ exists, $d_{i}$ must be the value assigned to $x$ by the consistent assignment. We can then add to $\pi$ the missing $X^{x_{i}, d_{i}}(w)$ in $\pi_{0}$ defined in the proof of Lemma A.2. After this extension, we get that there a homomorphism from $\pi$ to $I_{b}$ if and only if there is a satisfying assignment $\tau$ for $\varphi$, such that $\tau$ agrees with the assignments defined by the $X^{x_{i}, d_{i}}(w)$ that occur in $\pi$. Hence, a homomorphism from $\pi$ to $I_{b}$ exists due to the assumption of the lemma. And since the head variable of such an assignment must be mapped to $b$, we conclude that $b \in \pi(I)$. (And thus, $b \in \pi(I)$ held true before we added new atoms to $\pi$.)

\section{A.2 Proof of Lemma 5.4}

LEMMA 5.4. Let $\mathbf{H}$ be a hypothesis class that allows copying. The problem (H, CQ)-separability is coNP-hard already over the schema $\mathbf{S}_{0}$ and for two entities.

Proof. Let $G$ and $k$ be input for the maximum-clique problem, where $G$ is an undirected graph and $k$ is a natural number. The goal is to determine whether $G$ contains a subgraph isomorphic to $K_{k}$ (i.e., the complete graph over $k$ nodes).

We construct two new graphs, each with a set of nodes that is disjoint from that of $G$. The first graph is $G^{\prime}$, which is simply an isomorphic copy of $G$. The second is a copy $K$ of $K_{k}$.

We construct an instance $I$ over $\mathbf{S}_{0}$, as follows. We fix two entities $e_{G}$ and $e_{K}$, which are simply two distinct constants. The instance $I$ is the union of two subinstances $I_{G}$ and $I_{K}$, where:

- $I_{G}$ contains the fact $\eta\left(e_{G}\right)$ and, for every edge $\left\{v_{1}, v_{2}\right\}$ of $G$, the fact $G\left(v_{1}, v_{2}, e_{G}\right)$.

- $I_{K}$ contains the fact $\eta\left(e_{K}\right)$ and, for every edge $\left\{u_{1}, u_{2}\right\}$ of either $G^{\prime}$ or $K_{k}$, the fact $G\left(u_{1}, u_{2}, e_{K}\right)$.

We assume that the sets of nodes of $I_{G}$ and $I_{K}$ are disjoint. In particular, $I_{G}$ and $I_{K}$ consist of disjoint sets of facts. As said above, the instance $I$ is $I_{G} \cup I_{K}$. Note that $\eta_{I}$ consists of the two entities $e_{G}$ and $e_{K}$. The function $\lambda$ is defined by $\lambda\left(e_{G}\right)=1$ and $\lambda\left(e_{K}\right)=-1$. Hence, we have defined the training instance $(I, \lambda)$ over the entity schema $\mathbf{S}_{0}$.

We will prove there exists a statistic $\Pi$ in $C Q$ such that $(I, \lambda)$ is $\mathbf{H}$-separable w.r.t. $\Pi$ if and only if that $G$ does not contain a clique of size $k$.

The "if" direction. Suppose that $G$ does not contain any clique of size $k$. We will construct a statistic that separates $e_{G}$ from $e_{K}$. We define the following CQ.

$$
q(x) \leftarrow \bigwedge_{1 \leq i<j \leq k} G\left(y_{i}, y_{j}, x\right)
$$

Then $q(x)$ states that the graph associated with $x$ contains a clique of size $k$. Hence, we have that $e_{G} \notin q(I)$ and $e_{K} \in q(I)$, and the statistic $\Pi=(q)$ provides the required separation.

The "only if" direction. We now assume that $G$ contains a clique of size $k$. We will construct an endomorphism $\mu_{G K}: I \rightarrow I$ and an endomorphism $\mu_{K G}: I \rightarrow I$ such that $\mu_{G K}\left(e_{G}\right)=e_{K}$ and $\mu_{K G}\left(e_{K}\right)=e_{G}$. This suffices to prove the "only if" direction, as the existence of $\mu_{K G}$ and $\mu_{G K}$ implies that for every $q$ in CQ we have that $e_{G} \in q(I)$ if and only if $e_{K} \in q(I)$; therefore, every statistic $\Pi$ in CQ satisfies $\Pi\left(e_{G}\right)=\Pi\left(e_{K}\right)$. 
So, we complete the proof by constructing the endomorphisms $\mu_{G K}$ and $\mu_{K G}$. Let $C$ be a set of nodes in $G$ such that $|C|=k$ and the subgraph induced by $C$ is a clique. Let $\xi$ be an arbitrary one-to-one mapping from the nodes of $K$ to those in $C$, and let $\kappa$ be the mapping of the nodes of $G$ to their copies in $G^{\prime}$. We define $\mu_{G K}$ as follows.

$$
\mu_{G K}(x)= \begin{cases}e_{K} & \text { if } x \in\left\{e_{G}, e_{K}\right\} \\ x & \text { if } x \text { is a node of } G^{\prime} \text { or } K \\ \kappa(x) & \text { if } x \text { is a node of } G\end{cases}
$$

And we define $\mu_{K G}$ as follows.

$$
\mu_{K G}(x)= \begin{cases}e_{G} & \text { if } x \in\left\{e_{G}, e_{K}\right\} \\ x & \text { if } x \text { is a node of } G \\ \kappa(x) & \text { if } x \text { is a node of } G^{\prime} \\ \xi(x) & \text { if } x \text { is a node of } K\end{cases}
$$

It remains to prove that $\mu_{G K}$ and $\mu_{K G}$ are indeed endomorphisms, which is straightforward.

\section{A.3 Proof of Theorem 7.7}

THEOREM 7.7. Let $\mathbf{S}$ be an entity schema, and let $\Pi$ be a statistic in $\mathrm{CQ}$ such that $\pi_{i}^{c}$ is selective for all $\pi$ in $\Pi$.

1. If $\Pi$ has weak redundancy, then $\Pi$ is not affinely identifiable (hence, also not linearly identifiable).

2. If $\Pi$ has no weak redundancy, then $\Pi$ is linearly identifiable (hence, also affinely identifiable).

Proof. Throughout this proof we assume that $\Pi$ is represented as $\left(\pi_{1}, \ldots, \pi_{n}\right)$, where each $\pi_{i}$ is a CQ where $\pi^{c}$ does not contain in $\eta_{\mathbf{S}}(x)$.

Part 1. We assume that $\Pi$ has weak redundancy. Let $I$ be an instance over $\mathbf{S}$. We need to show that $\llbracket \Pi^{I} \rrbracket$ has an affine column dependency. Let $C_{1}, \ldots, C_{n}$ be the columns of the matrix $\llbracket \Pi^{I} \rrbracket$.

Consider first the case where there are two or more equivalences among $\pi_{1}^{c}, \ldots, \pi_{n}^{c}$, then there are two options.

1. There are $i<j<k$ such that $\pi_{i}^{c}, \pi_{j}^{c}$ and $\pi_{k}^{c}$ are equivalent. If at least two of $\pi_{i}^{d}, \pi_{j}^{d}$ and $\pi_{k}^{d}$ are true, then at least two of $C_{i}, C_{j}$ and $C_{k}$ are the same (since the connected parts are equivalent). If at least two of $\pi_{i}^{d}$, $\pi_{j}^{d}$ and $\pi_{k}^{d}$ are false, then again at least two of $C_{i}, C_{j}$ and $C_{k}$ are the same (all -1$)$. Hence, in any case, $M$ has an affine column dependency.

2. There are four distinct indices $i<j$ and $k<l$ such that $\pi_{i}^{c}$ is equivalent to $\pi_{j}^{c}$ and $\pi_{k}^{c}$ is equivalent to $\pi_{l}^{c}$. If at least two of $\pi_{i}^{d}, \pi_{j}^{d}, \pi_{k}^{d}$ and $\pi_{l}^{d}$ are false, at least two of $C_{i}, C_{j}, C_{k}$ and $C_{l}$ are the same. And if at most one of $\pi_{i}^{d}, \pi_{j}^{d}, \pi_{k}^{d}$ and $\pi_{l}^{d}$ is false, then either $C_{i}=C_{j}$ or $C_{k}=C_{l}$. Hence, in any case, $M$ has an affine column dependency.

Consider now the case where there is exactly one equivalence among the $\pi_{1}^{c}, \ldots, \pi_{n}^{c}$. So, we assume now the feature queries $\pi_{i}$ and $\pi_{j}$ as defined in Definition 7.6. In particular, the only equivalence among $\pi_{1}^{c}, \ldots, \pi_{n}^{c}$ is that between $\pi_{i}^{c}$ and $\pi_{j}^{c}$. We consider several options.

1. If both $\pi_{i}^{d}$ and $\pi_{j}^{d}$ are false in $I$, then $C_{i}=C_{j}$ and $M$ has an affine column dependency.

2. Similarly, if both $\pi_{i}^{d}$ and $\pi_{j}^{d}$ are true in $I$, then $C_{i}=C_{j}$ (since $\pi_{i}^{c}$ and $\pi_{j}^{c}$ equivalent), and then again $M$ has an affine column dependency.

3. If $\pi_{i}^{d}$ is false and some $\pi_{l}^{b}$ is false for $l \neq i$, then $C_{i}=C_{l}$ and $M$ has an affine column dependency.

4. Similarly, if $\pi_{j}^{d}$ is false and some $\pi_{l}^{b}$ is false for $l \neq j$, then has an affine column dependency.

Eliminating all of the above cases, we have that exactly one of $\pi_{i}^{d}$ and $\pi_{j}^{d}$ is false in $I$. Suppose, without loss of generality, is $\pi_{i}^{d}$ is false in $I$. So $\pi_{l}^{b}$ is true for every $l \neq i$. But this is impossible, since $\pi_{i}^{d}$ is logically entailed by the conjunction of the $\pi_{l}^{b}$ for $l \neq i$.

Part 2. We assume that $\Pi$ has no weak redundancy, and we will construct an instance $I$ over $\mathbf{S}$ such that $\llbracket \Pi^{I} \rrbracket$ has degree $n$. We consider two cases.

In the first case, we assume that no two CQs among $\pi_{1}^{c}, \ldots, \pi_{n}^{c}$ are equivalent. In this case, we proceed as in the proof of Theorem 7.2. In particular, note that we satisfy each $\pi_{i}^{d}$ by facts of an active domain that is disjoint from that of the remaining database. The reader can verify that, due to this disjointness, that proof holds in this case as well.

In the second case, we assume (without loss of generality) that there is exactly one equivalence among $\pi_{1}^{c}, \ldots, \pi_{n}^{c}$, and it is between $\pi_{n-1}^{c}$ and $\pi_{n}^{c}$. Moreover, we assume that $\pi_{n}^{d}$ is not logically entailed by the conjunction of the $\pi_{l}^{b}$ for $l<n$. In this case, we construct the instance $I$ as follows. For $i=1, \ldots, n-1$, we define $I_{i}$ as a canonical instance for $\pi_{i}$, using a fresh set of constants. For $i=1, \ldots, n-1$, let $e_{i}$ be the entity introduced for $\pi_{i}$. For $\pi_{n}$ we do not introduce any new fact, besides the new entity $e_{n}$. Since we have used pairwise-disjoint canonical instances, we have that $\pi_{n}^{d}$ is false in $I$. We conclude by showing that $\llbracket \Pi^{I} \rrbracket$ is linearly column independent. Let $C_{1}, \ldots, C_{n}$ be the columns of the matrix $\llbracket \Pi^{I} \rrbracket$, and let $r_{1}, \ldots, r_{n}$ be its rows. Then we have the following.

- $C_{n}$ consists of only -1 , because is $\pi_{n}$ is false in $I$.

- $r_{n}$ consists of only -1 , because no $\pi_{i}^{c}$ is equivalent to $\eta_{\mathbf{S}}$ (hence, $e_{n} \notin \pi_{i}^{c}(I)$ and $\left.e_{n} \notin \pi_{i}(I)\right)$.

So we will now continue like the proof of Theorem 7.2, but restrict ourselves to the rows $r_{1}, \ldots, r_{n-1}$, and use $r_{0}=-r_{n}$. So, we have $n$ rows that are a linear combination of the rows of $M$. For $1 \leq i<n-1$ we have that $r_{i}^{\prime}=r_{i}-r_{n}$ is such that the $n$th column is 0 , and so, all the arguments of that proof still hold and we have that $a_{i}=0$ for $i=1, \ldots, n-1$. And of course, we then have $a_{n}=0$ since the $n$th element of $r_{0}$ is nonzero. 